\title{
Versuche zur Erfassung der vertikalen Verteilung von Organismen und chemischen Substanzen im Grundwasser von Talauen und Terrassen; Methoden und erste Befunde
}

von

\author{
Siegfried HUSMANN*
}

\author{
ABSTRACT \\ Investigations into the vertical distribution of organisms and \\ chemical substances in the groundwater in valleys and \\ terraces; methods and first results.
}

In the alluvial ground of the river Fulda valley and in the diluvial terrace of the river Weser assortments of tubes of various lengths were sunk into sandy and gravelly underground to bring to light groundwater of different depths.

The installation of these groundwater pump stations was effectuated by two different methods: 1. with the aid of an apparatus for bringing down bore-holes, 2. by ramming in the pump tubes with the aid of a pneumatic hammer.

The first biological and chemical investigations in these subterranean water research stations indicated that the vertical distribution of groundwater organisms and chemical substances in special cases may depend on the nature of subterranean water currents and the infiltration of polluted water into the sandy and gravelly underground of valleys and terraces.

\section{INHALT}

I. Einleitung . . . . . . . . . . . . . . . . . . . 272

II. Die Untersuchungsstellen . . . . . . . . . . . . . . . . . . 273

1. Die Station "Weserterrasse" bei Liebenau . . . . . . . . . . . . . 273

2. Die Station "Fulda-Aue" bei Fulda . . . . . . . . . . . . . 278

III. Methodik . . . . . . . . . . . . . . . . . . . . . . . . . . 282

1. Bau der Peilrohr-Stationen . . . . . . . . . . . . . . . 282

a) Abteufung der Peiirohre mittels Ventilbohrerverfahrens . . . . . . 283

b) Einrammen der Peilrohre mittels Presslufthammers . . . . . . . 285

2. Proben - Entnahme . . . . . . . . . . . . . . . 285

a) Substrat-Proben . . . . . . . . . . . . . . . . . . 286

b) Wasserproben für biologische Untersuchungen . . . . . . . . 287

c) Wasserproben für chemische Untersuchungen . . . . . . . . . 288

\footnotetext{
* Limnologische Fluss-Station des Max-Planck-Institutes für Limnologic, D 6407 Schlitz/Hessen
} (West-Deutschland). 
3. Untersuchungsmethoden . . . . . . . . . . . . . . . . . . 289

a) Granulometrische Substrat-Analyse . . . . . . . . . . . . . . . . 289

b) Chemische Wasseruntersuchung . . . . . . . . . . . . . . . . . . 290

c) Biologische Wasseruntersuchung . . . . . . . . . . . . . . . . . . 290

IV. Erste Ergebnisse und Probleme . . . . . . . . . . . . . . . . . . . . . 290

1. Das chemisch-biologische Vertikalprofil im Grundwasser der Weser-

Niederterrasse bei Liebenau . . . . . . . . . . . . . . . . . . . 290

2. Das chemisch-biologische Vertikalprofil im Grundwasser der Fulda-

Aue bei Fulda . . . . . . . . . . . . . . . . . . . . . . . . . 293

V. Die ökologisch-hygienische Aussage der Befunde . . . . . . . . . . . 295

VI. Dank und Hinweise . . . . . . . . . . . . . . . . . . . . . . 299

VII. Zusammenfassung . . . . . . . . . . . . . . . . . . . . . . . . . . 299

Summary ....................... 300

VIII. Literatur . . . . . . . . . . . . . . . . . . . . . 300

\section{EINLEITUNG}

Eine oft arten- und individuenreiche Grundwasserfauna besiedelt deutlich bevorzugt die wasserführenden Lückensysteme in Ablagerungen von Sanden und Kiesen im Untergrund von Talauen und Terrassen. Grundwässer in Sand und Kies werden daher als Hauptökotope blinder und pigmentloser Wassertiere zum wesentlichen Untersuchungsobjekt der ökologischen Grundwasserforschung.

Da die von Grundwasser erfüllten Lückensysteme in sandigkiesigen Lockergesteinen natürliche Speicher guten Trinkwassers darstellen können, erlangt das interstitielle Grundwasser auch seitens der Wasserwirtschaft besondere Bedeutung; und weil naturgemäss sandigkiesige Gerölle ganz erheblich durch Infiltrationen aus verunreinigten Gewässern der Erdoberfläche be"einflusst" werden können, wächst heute mit steigender Wassersorge auch die Notwendigkeit einer konzentrierten hygienischen Überwachung intergranularer Grundwässer: Subterranökologie und Grundwasserhygiene begegnen einander also am gleichen Forschungsobjekt (vgl. Husmann 1971a); und beide Arbeitsrichtungen dienen praktisch auch der Gewinnung von Erkenntnissen zur Förderung einer notwendigen Sicherung der unterirdischen wasserführenden Umwelt. Untersuchungsbereiche einer solchen ökohygienisch orientierten Stygologie liègen beispielsweise in Schotterbetten verunreinigter Fliessgewässer, aber auch im ökologisch gestörten Grundwasser sandigkiesiger Ablagerungen im Untergrund von Talauen und Terrassen.

Über horizontale Infiltrationswege von Abwasserschleiern im Grundwasser sandigkiesiger Ablagerungen liegen bereits Untersuchungsbefunde vor (Farkasdi, Golwer, Knoll, Mathess u. Schneider 1969; Nöring, Farkasdi, Golwer, Knoll, Mathess u. Schneider 1968; Golwer, Mathess u. Schneider 1969; Golwer, Knoll, Mathess, Schneider u. Wallhäuser 1972; Jaag 1952; Rössler 1951; Zwitting 1964; siehe auch Literaturverz. dieser Arbeiten). 
Die ökologische Untersuchung vertikaler Einflüsse versickernder Wasserverunreinigungen auf Wasserchemismus und Mehrzellerfauna in LockergesteinsLückensystemen wurde meines Wissens zuerst durch Arbeiten an Langsamfiltern in Wasserwerken vorgenommen (Husmann 1958, 1961, 1966). Diese Sandfilter erwiesen sich dabei als besonders aussagekräftige "Biotopmodelle der experimentalökologischen Grundwasserforschung” (Husmann 1968).

Bei diesen Filtersand-Untersuchungen zeigten sich intergranulare Erscheinungen, die sich in ihrer Grundtendenz auch bei späteren Untersuchungen in Schotterbetten von Fliessgewässern ergaben: wesentliche Prozesse der Sandfiltration verlaufen in den oberen, freiwassernahen Zentimetern sowohl innerhalb von Filtersandschichten als auch in Schotterbettgeröllen (Husmann 1971).

Diese Befunde regten dazu an, nun auch die bisher noch nicht bekannte vertikale Verteilung von Organismen und chemischen Substanzen im Grundwasser von Talauen und Terrassen zu untersuchen, und zwar von Peilrohrstationen aus, die es ermöglichen, Grundwasser aus verschiedenen Tiefen zutage zu fördern.

\section{DIE UNTERSUCHUNGSSTELLEN}

Die Peilrohrstationen wurden rings um Grundwasserbeobachtungsrohre geschlagen, aus denen bei früheren ökologischen Grundwasseruntersuchungen eine besonders interessante Subterranfauna bereits zutage gepumpt worden war. Die beiden bisher schon in Betrieb genommenen Untersuchungsstationen liegen im Weser-System (Karte 1). Die zuerst angelegte steht in der Niederterrasse der Unterweser (Karte 2), die andere in der Fulda-Talaue (Karte 3).

\section{Die Station "Weserterrasse" bei Liebenau}

Standortbestimmend wurde das Peilrohr XIId des Wasserwerkes Liebenau der Harzwasserwerke des Landes Niedersachsen, eine Fundstelle besonders seltener stygobionter Grundwassertiere. Über einige interessante Arten aus P.XII d wurde bereits berichtet (Husmann 1962, 1964, 1968). An Bathynellen (Crustacea, Syncarida) aus diesem Peilrohr werden zur Zeit im Zoologischen Institut der Universität Erlangen anatomische Untersuchungen ausgeführt.

Die Station XIId steht in einer Lehmgrube, in der infolge unrechtmässiger Deponierung von Schutt das Landschaftsbild erheblich verunstaltet wird.

Die Anlage der Peilrohrstation gab daher Anlass, zum Schutz der reliktären, seltenen Grundwassertiere einen Geländestreifen von 3239 qm Flächengrösse als Naturdenkmal zu sichern (XII. Nachtragsverordnung zur Sicherung von Naturdenkmalen, Amtsblatt für den Regierungsbezirk Hannover v. 13. VIII. 1970, S. 271).

Noch vor Inkrafttreten dieser Schutzverordnung wurde um das Peilrohr XIId herum Fäkalien-Schlamm abgelagert, der dort jetzt in einer $15-20 \mathrm{~cm}$ mächtigen erdigen Schicht den natürlichen Boden bedeckt.

Dieser zunächst als überaus störend angesehene, aus einer Kläranlage entstammende Infiltrationsherd gab den Untersuchungen eine neue Richtung: sie wurden 


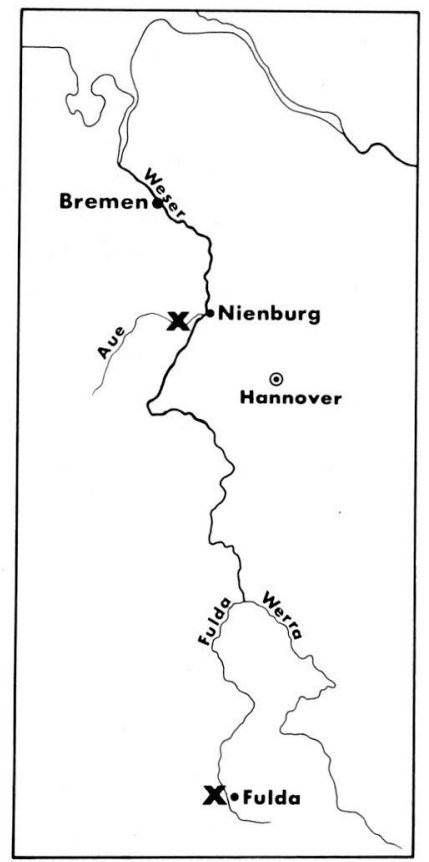

Karte 1.

Geographische Lage der Peilrohr-Stationen an Fulda und Unterweser.

Karte 2.

Geologische Karte der Umgebung von Peilrohr-Station XIId (Liebenau); nach Manuskriptkarte Dr. W. Straute, Niedersächsisches Amt für Bodenforschung, Hannover. Mit Lagebezeichnung der Station und der Flusswasser-Entnahmestellen an Weser $(\mathrm{W} \rightarrow)$ und Aue $(\mathrm{A} \rightarrow)$. Legenden-Erläuterung: 17: noch nicht kartiert; 16: Anmoor über fluviatilem Sand der zweiten Holozänstufe (Marklohe-Komplex; 15: Anmoor über Auelehm des Esdorfer Komplexes; 14: Auelehm der zweiten Holozänstufe; 13: Auelehm über Fluss-Sand der ersten Holozänstufe (Estorfer Komplex); 12: Auelehm der ersten Holozänstufe (Estorfer Komplex); 11: Fluss-Sand der ersten Holozänstufe über Sand und Kies der Niederterrasse; 10: Flugsand; 9: Flugsand über Sand und Kies der Niederterrasse; 8: Flugsand in flächenhafter Verbreitung über Kies (Drenthe); 7 : Hochflutlehm der Niederterrasse über Sand der Niederterrasse; 6: Sand und Kies der Niederterrasse; 5: Sand der Niederterrasse; 4: Geschiebedecksand über Geschiebelehm (Drenthe); 3: Geschiebedecksand über glazifluviatilem Sand (+ Kies) des Drenthe-Stadiums; 2: Glazifluviatiler Sand (Drenthe-Stadium); 1: Glazifluviatiler Kies (Drenthe-Stadium). (Nach Husmann 1968a; mit Ergänzungen versehen). 


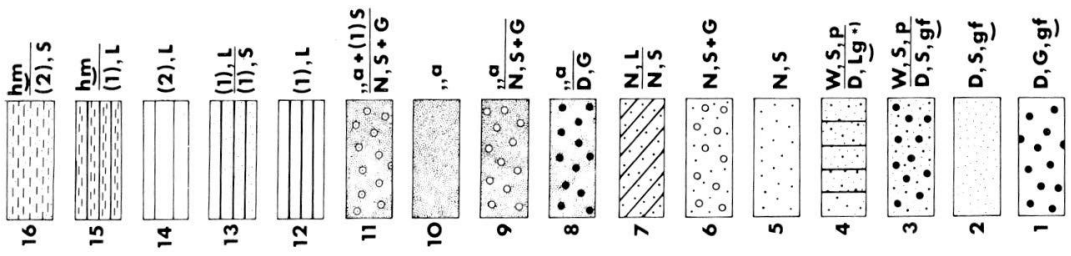

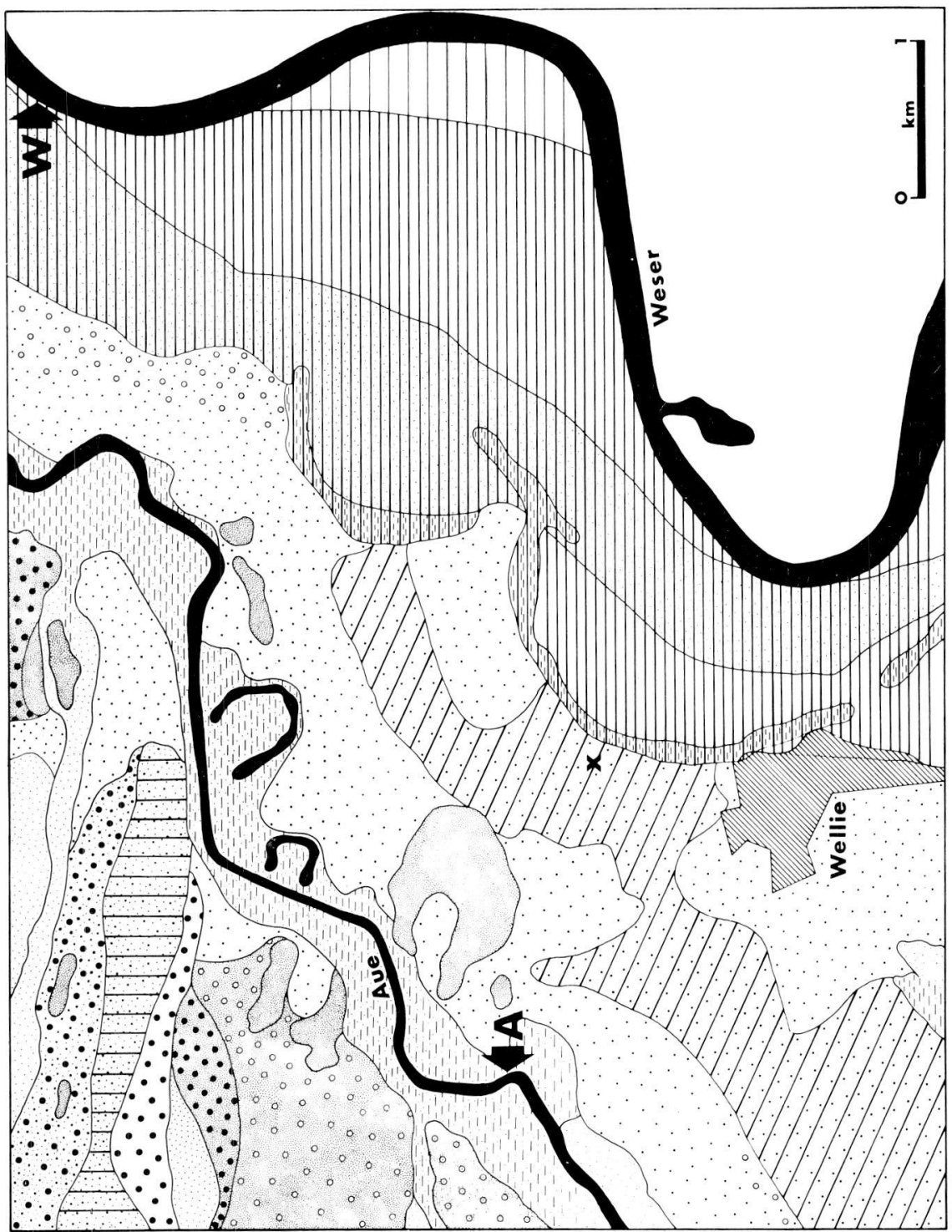




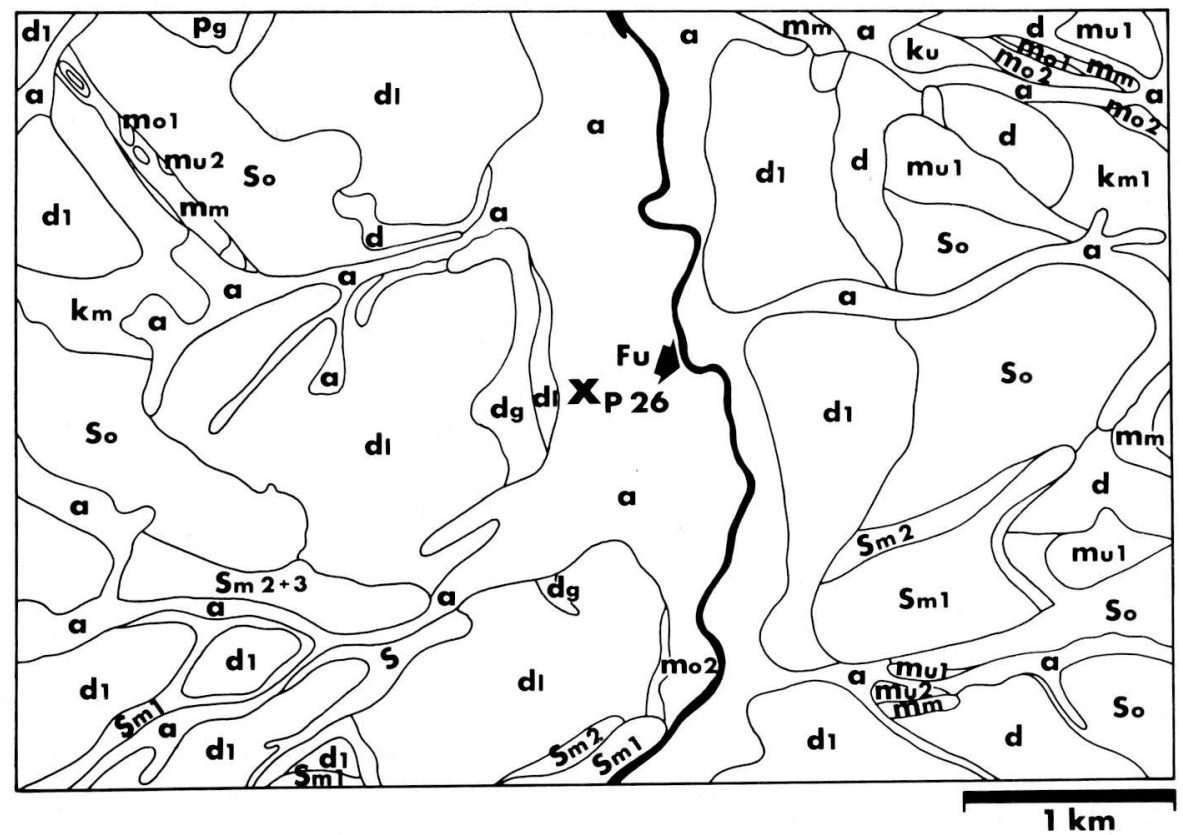

Karte 3.

Geologische Karte der Umgebung von Peilrohrstation Fulda-Aue (P. 26) mit Lagebezeichnung von Station und Flusswasser-Entnahmestelle $(\mathrm{Fu} \rightarrow)$.

Erläuterung der Signaturen: $a$ : Alluvialer Auelehm mit Kies und Sand; d: Diluvialer Lehm; 1 : Diluv. Schotter, vermischt mit Sand und Lehm; $d g$ : Schotter, vorwiegend Buntsandstein und Quarzgeröll; $d 1$ : Löss und Lösslehm; bp: Pliozäne Tone und Sande; $k o:$ Quarzite und Letten des Oberen Keuper (Rät); $k m$ : Mergel, Dolomite und Steinmergel des Gipskeuper; $k u$ : Kohlenkeuper; Sandsteine, Letten, Grenzdolomit; mu1: Unterer Muschelkalk (Wellenkalk, Kalkschiefer); mu2: Oberer Wellenkalk, Kalkschiefer; $m m$ : Mittlerer Muschelkalk (Dolomitischer Kalk, Mergel u. Wellenkalk; mol: Oberer Muschelkalk (Trochitenkalk); mo2: Oberer Muschelkalk (Ceratiten-Schichten, plattige Kalke und Mergel m. Ceratites nodosus; sm1: Hauptbuntsandstein; $s m 2$ : Chirotheriensandstein; $s m 2$;3: Chirotherien- u. Bausandstein; so: Oberer Buntsandstein (Röt).

zu einem Gross-Feldversuch zur Erfassung der vertikalen Infiltration von Verunreinigungsstoffen ins Grundwasser; und die oben erwähnten Massnahmen zur Sicherung der Landschaft schirmen zugleich diese Arbeiten gegen spätere ökologische Einflüsse ab, die durch neue Zufuhr von Unrat und Müll in der Umgebung der Peilrohrstation immerhin zu erwarten waren. Mit anderen Worten: die Infiltrationsbedingungen im Bereich der Untersuchungsstation "Weserterrasse" haben grösstmögliche Konstanz!

Da im engeren Bereich der Untersuchungsstelle der die Erdoberfläche bildende Hochflutlehm (Karte 2) abgebaut worden ist, stehen die Peilrohre der Station abgesehen von der Klärschlammauflage - im Sand der Niederterrasse, dem naturgegeben Liegenden des Hochflutlehms. Infiltrationen von der Erdoberfläche her 

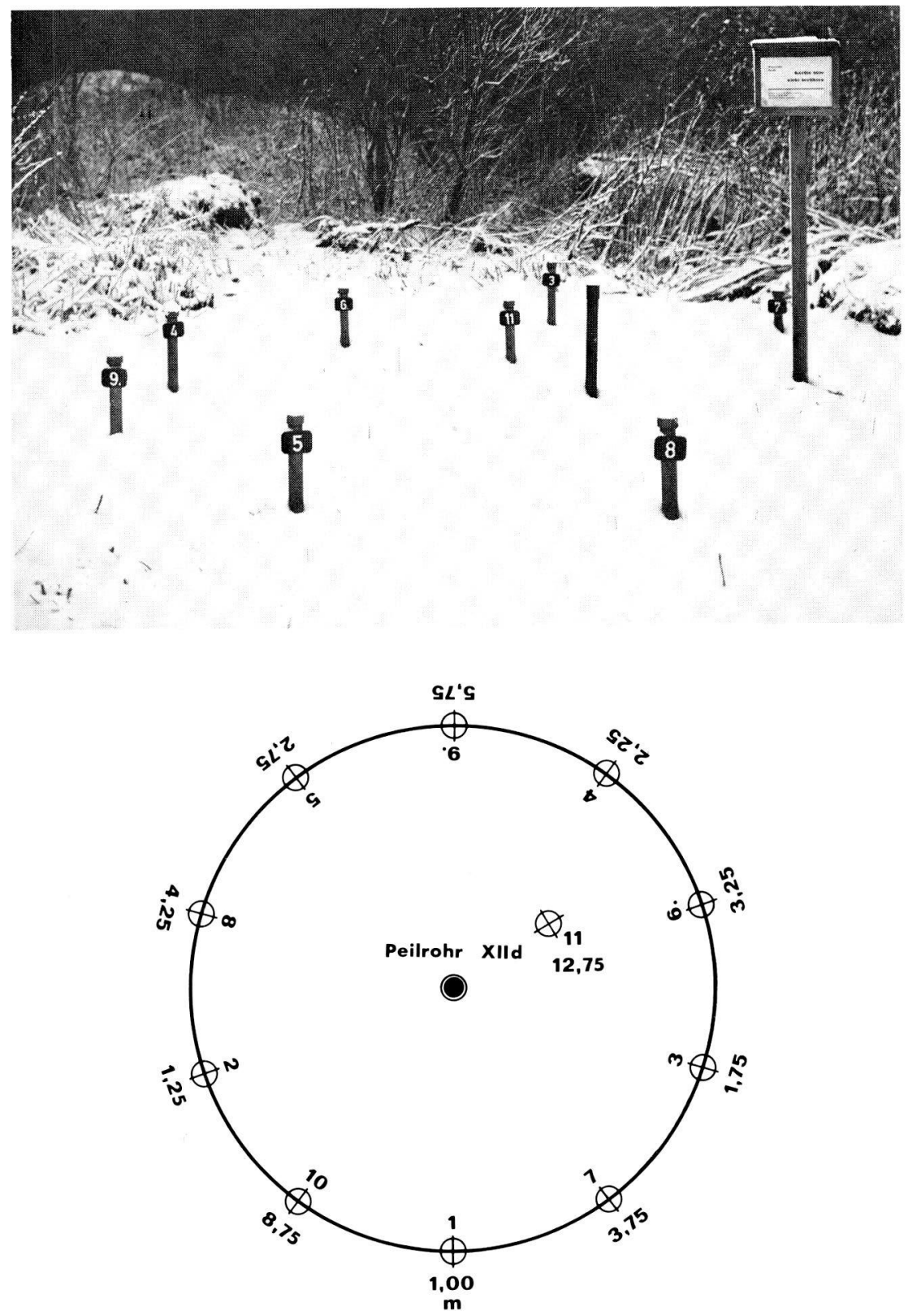

Abb. 1. a) Peilrohr-Station Liebenau XIId; b) P.-St. XIId, Lageplan der einzelnen Peilrohre mit Angaben zur Tiefenlage der Einlauf-Offnungen. 
stehen im Bereich von Station XIId also relativ freie Versickerungswege offen. Das gilt auch für alle von Natur aus sandigkiesigen Regionen der umliegenden Landschaft (Karte 2); ganz abgesehen von den offenen horizontalen Infiltrationswegen in grundwasserführenden Lockergesteins-Ablagerungen des Untergrundes.

Da die P.-Station gerade im Mündungswinkel gelegen ist, der von der Weser und ihrem Nebenfluss Aue gebildet wird, musste von vornherein mit der Möglichkeit subterraner Horizontal-Infiltration aus zumindest einem dieser Flüsse gerechnet werden. Daher wurden von bestimmten Entnahmestellen aus (Karte 2) Wasserproben aus beiden Flüssen an den jeweiligen Untersuchungstagen mit entnommen.

Die 11 Peilrohre der Station reichen unter der Eroberfläche bis in eine Tiefe von 12,75 m hinab (Anordnung der Rohre und Tiefenlagen der Grundwasser-Einlauföffnungen zeigt Abb. 1).

Das zum Einbringen der Rohre in den Untergrund der Niederterrasse angewendete Verfahren (vgl. S. 283) ermöglichte es, Substratproben genau aus den Tiefenlagen zu entnehmen, in denen sich die Grundwasser-Einlauföffnungen der einzelnen Rohre befinden. Granulometrische Analysen (vgl. S. 289) konnten daher genauen Aufschluss über die Korngrössen-Zusammensetzung der jeweils angezapften grundwasserführenden Substrate geben.

Die Ergebnisse dieser Korngrössen-Analysen zeigen, dass in allen erfassten Tiefenlagen Substrate sandiger Natur (Psammite) das Volumen der grundwasserführenden Interstitialräume bestimmen (Abb. 2), da psephitische Substratkomponenten einen derart geringen Anteil stellen, dass sie hohlraumgestaltend überhaupt nicht wirksam werden können (vgl. Husmann 1966, p. 234).

\section{Die Station "Fulda-Aue" bei Fulda}

Die individuenreiche Biocoenose des in der Talaue der Fulda nahe der Stadt Fulda stehenden Peilrohres 26 (Karten 1 u. 3) gab Anlass, rings um dieses Rohr herum eine zweite Peilrohrstation (Abb. 3) einzurichten.

Die Peilrohre entnehmen hier das Grundwasser sandigkiesigen Substraten des Alluviums, die von über 2 m mächtigen Ablagerungen von Schluff, Sand und Ton überlagert werden. Wenn auch die Landschaft ringsum an der Erdoberfläche nur wenig infiltrationsfördernde Lockergesteine aufweist und daher also Versickerungsbereiche im Vergleich zur Umgebung der Station "Weserterrasse", jedenfalls flächenmässig, von geringerer Bedeutung sind (Karte 3), so könnte hier doch unter Umständen ein anderer Faktor Infiltrationen in den Untergrund der Talaue hinein fördern: das von den Buntsandstein-, Muschelkalk- und Keuper-Hängen beiderseits der Fulda in die sandigkiesigen Ablagerungen der Talaue einmündende Grundwasser! Aber auch der Grundwasserstrom des Fuldabettes dürfte mit dem uferfernen Talauengrundwasser in Verbindung stehen. Daher wurde auch an der Fulda eine Wasserproben-Entnahmestelle festgelegt (Karte 3).

Die Peilrohre dieser Station reichen bis in eine Tiefe von $4 \mathrm{~m}$ herab. Auf Grund des angewendeten Verfahrens beim Einbringen der Rohre in den Untergrund (vgl. S. 285) liegen für diese Untersuchungsstelle keine eindeutigen Befunde über die Substratbeschaffenheit in der Tiefenlage der Grundwasser-Einlauföffnungen vor. 


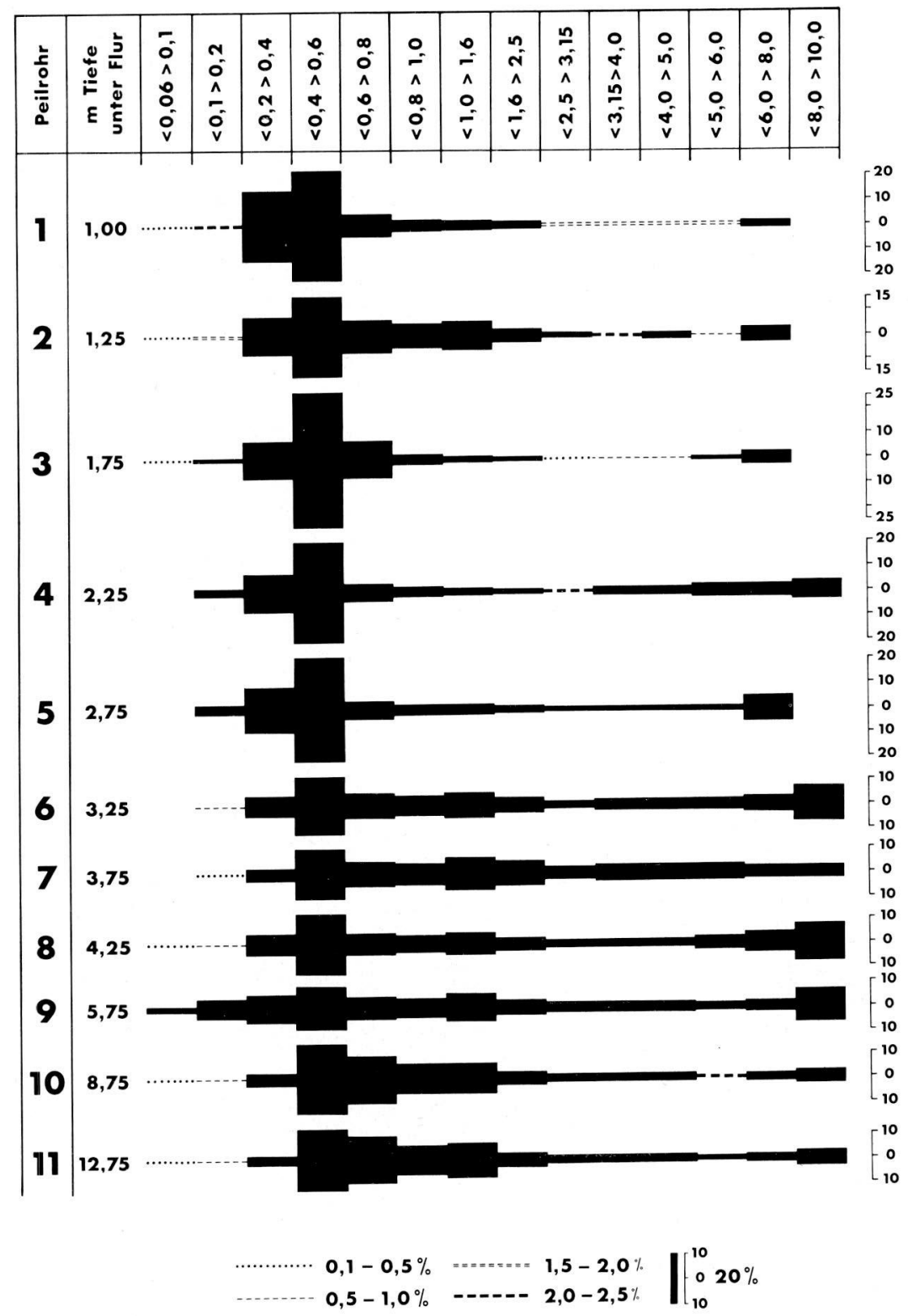

Abb. 2. Die prozentuale Verteilung der Korngrössen in den Substraten der erfassten Tiefenlagen von Peilrohr-Station Liebenau XIId. 
Hierzu gibt jedoch eine Bohrung Aufschluss, die 1962 von Herrn Prof. Dr. F. Preul, Niedersächsisches Landesamt für Bodenforschung, Hannover, ausgeführt und ausgewertet wurde (Abb. 4), denn dieses Bohrloch wurde speziell zum Einbau des Peilrohres 26, im Verlauf hydrogeologischer Untersuchungen (Preul 1962), angelegt.

Wie Abb. 4 zeigt, entnehmen die Peilrohre der Station Fu/26 Interstitialwasser aus Lockergesteinsablagerungen sowohl psammitischer als auch psephitischer Natur.

Für eine ökologische Beurteilung der Angaben über die Zusammensetzung der grundwasserführenden Substrate ist die oben schon erwähnte Verschiedenartigkeit der zum Einbau der Peilrohre verwendeten Verfahren von besonderer Bedeutung. Auf diese Verfahrensweise wird im folgenden noch näher einzugehen sein.

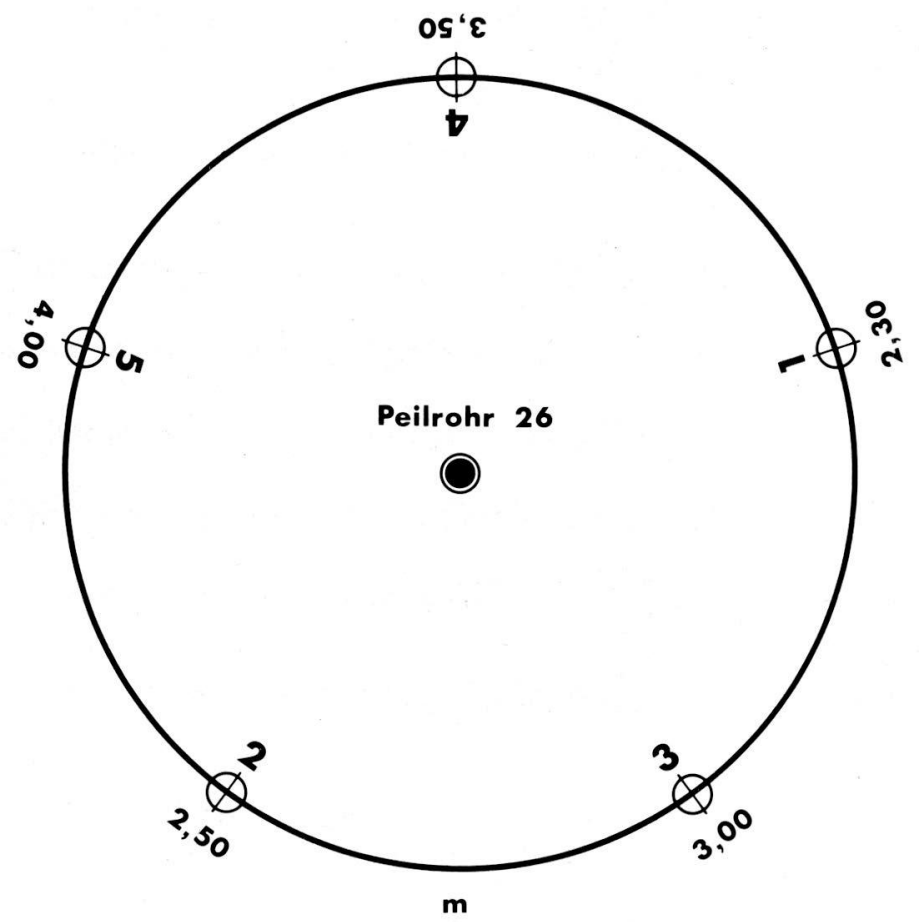

Abb. 3. Lageplan der einzelnen Peilrohre der Station Fulda-Aue (P. 26) mit Angaben zur Unter-Flur-Tiefenlage der Einlauföffnungen. 


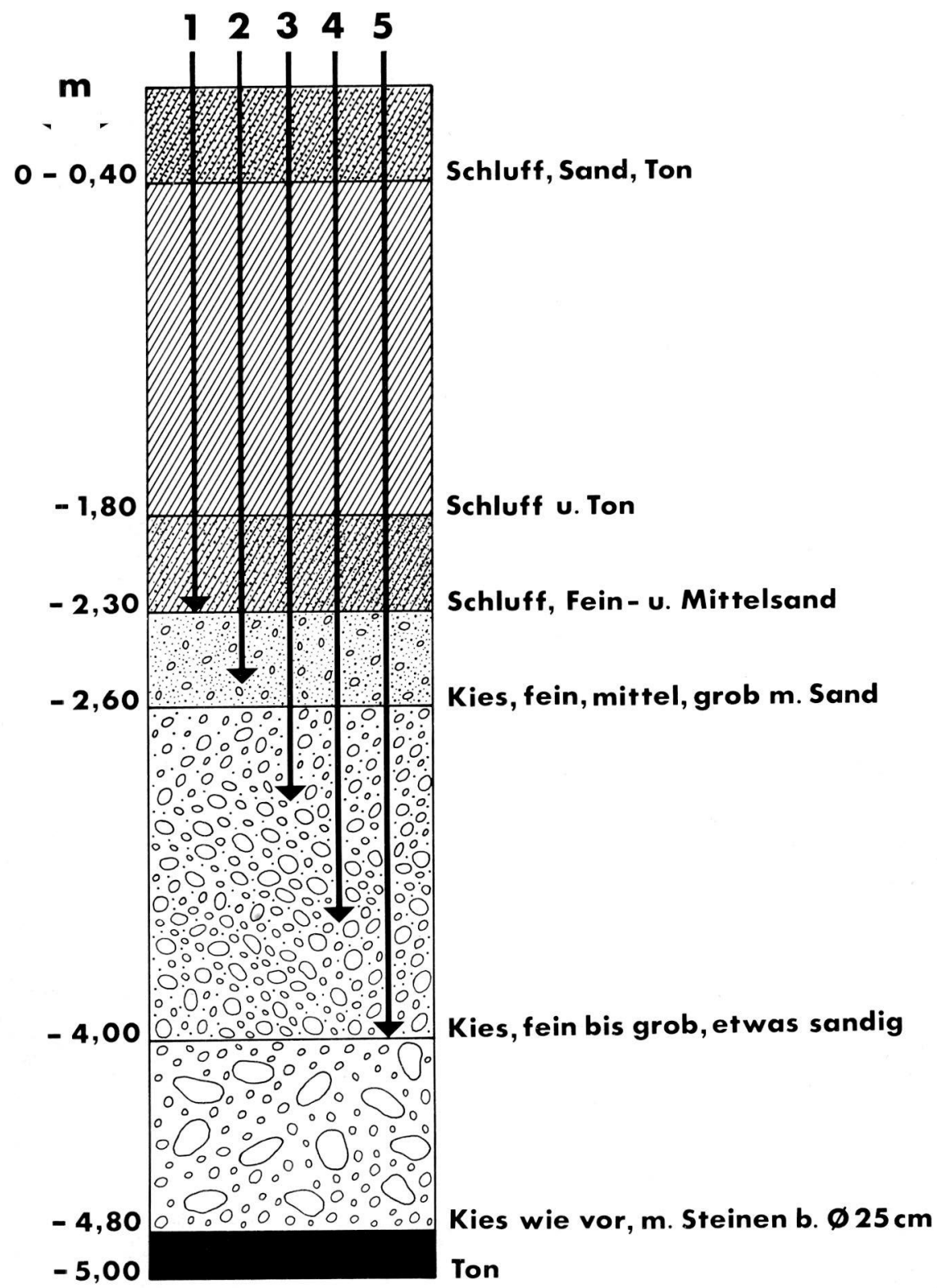

Abb. 4. Schichtenfolge im Untergrund der Fulda-Aue bei P.-Station 26 mit dort abgeteuften Peilrohren. 


\section{METHODIK}

Als Peilrohre wurden verzinkte Eisenrohre $(\phi 47 \mathrm{~mm})$ verwendet, deren im Untergrund stehendes perforiertes Rohrende (Abb. 8,b) zugeschweisst oder mit einer massiven Schlagbolzenspitze versehen wurde, je nach dem angewandten Verfahren beim Einbringen der Rohre in den Untergrund.

\section{Bau der Peilrohr-Stationen}

Da die Peilrohr-Stationen in Zusammenarbeit mit Wasserwerken eingerichtet wurden (vgl. S. 273), lag es nahe, den Einbau der Rohre mit den jeweils gegebenen technischen Möglichkeiten zu bewerkstelligen:
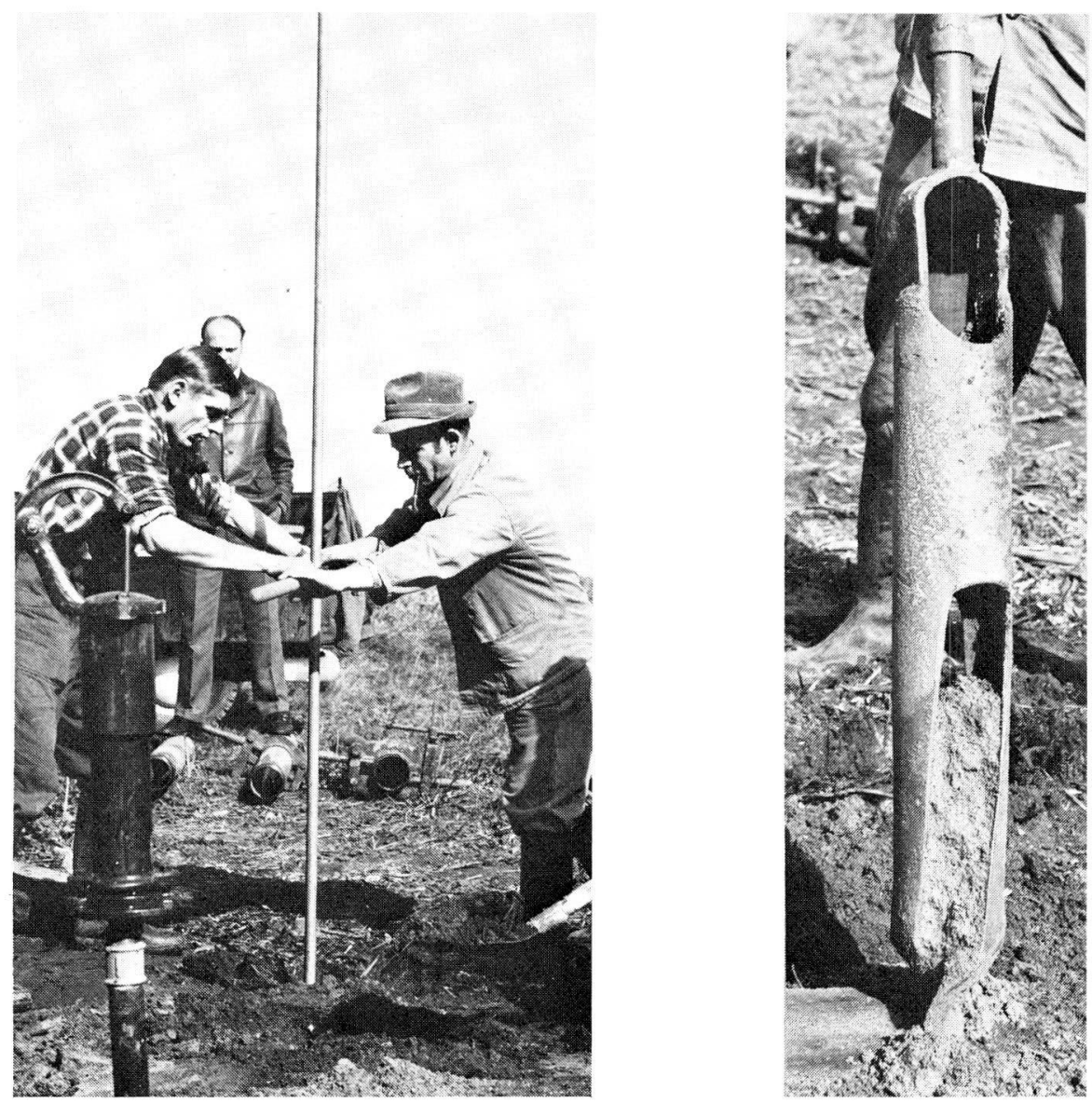

Abb. 5. a) Bohrung mit Löffelbohrer; b) Gezogener Löffelbohrer mit Substrat. 


\section{a) Abteufung der Peilrohre mittels Ventilbohrer-verfahrens}

Die Unterweser-Station wurde mit Hilfe eines Handbohrers und einer VentilbohrerEinrichtung angelegt (Abb. 5,6,7,8). Für jedes Peilrohr dieser Station wurde zunächst ein Bohr-Rohr (innerer $\phi: 120 \mathrm{~mm}$, äusserer $\phi 130 \mathrm{~mm}$ ) abgeteuft (Abb. 6, 8), um auf diese Weise ein Zusammenrutschen der durchteuften Lockergesteine zu verhindern. Zur Ausführung der Bohrarbeiten wurde bis zum Erreichen des Grundwasserspiegels ein Löffelbohrer (vgl. Damrath, 1963, S. 37 u. Abb. 37, 4) verwendet (Abb. 5, a, b). Das grundwasserführende Substrat hingegen wurde mit Hilfe eines Ventilbohrers (äusserer $\phi: 100 \mathrm{~mm}$ ) zutage gefördert (Abb. 6, 7; vgl. Damrath 1963, S. 37 u. Abb. 37, 4). Nach Erreichen der endgültigen Bohrtiefe wurde dann jeweils das betreffende Peilrohr zentrisch in das Bohrrohr eingebracht (Abb. 8, a). Zug um Zug folgte darauf das Herausziehen des Bohrrohres aus dem Bohrloch und

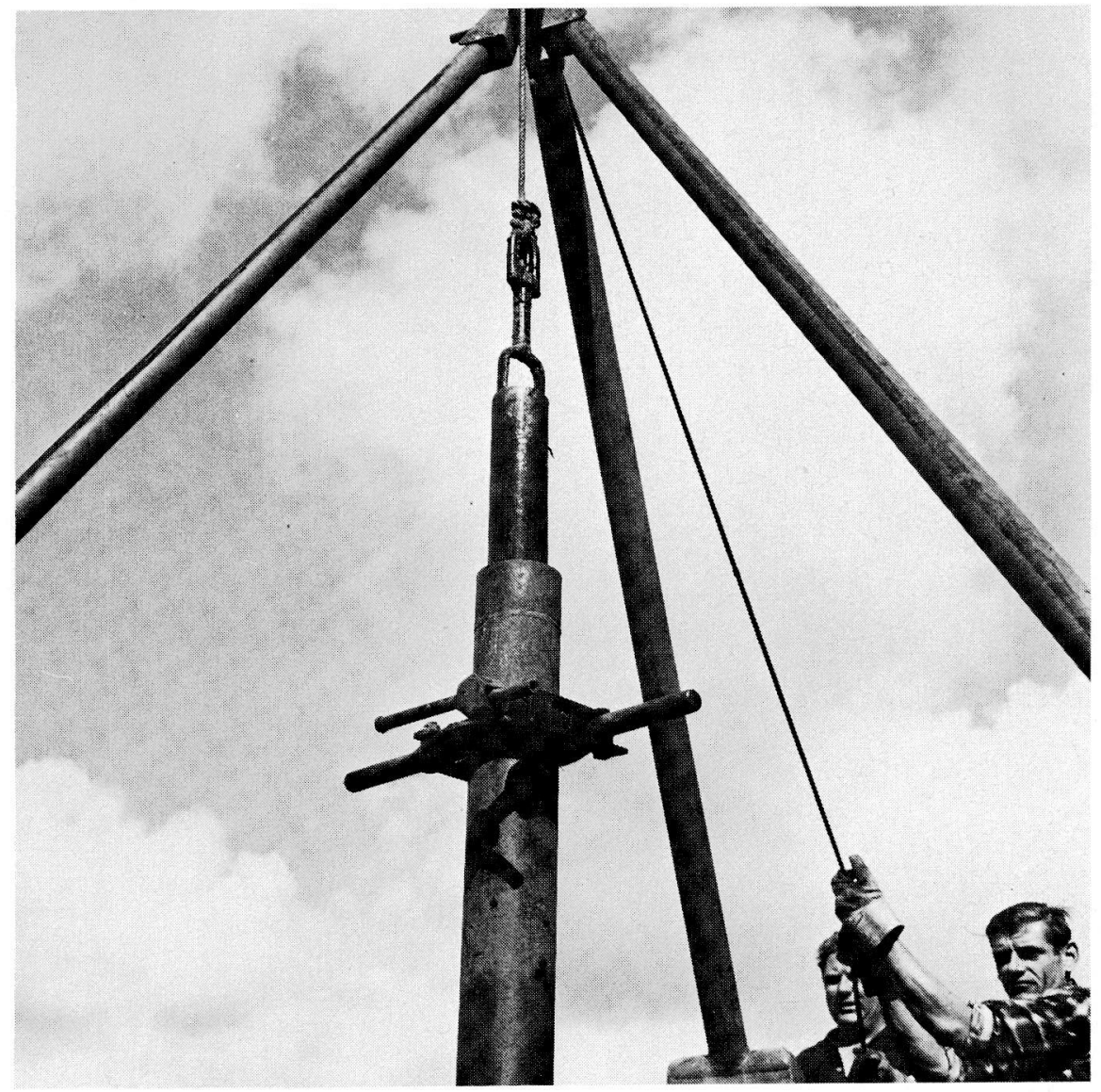

Abb. 6. Bohrgerüst mit Ventilborer. 


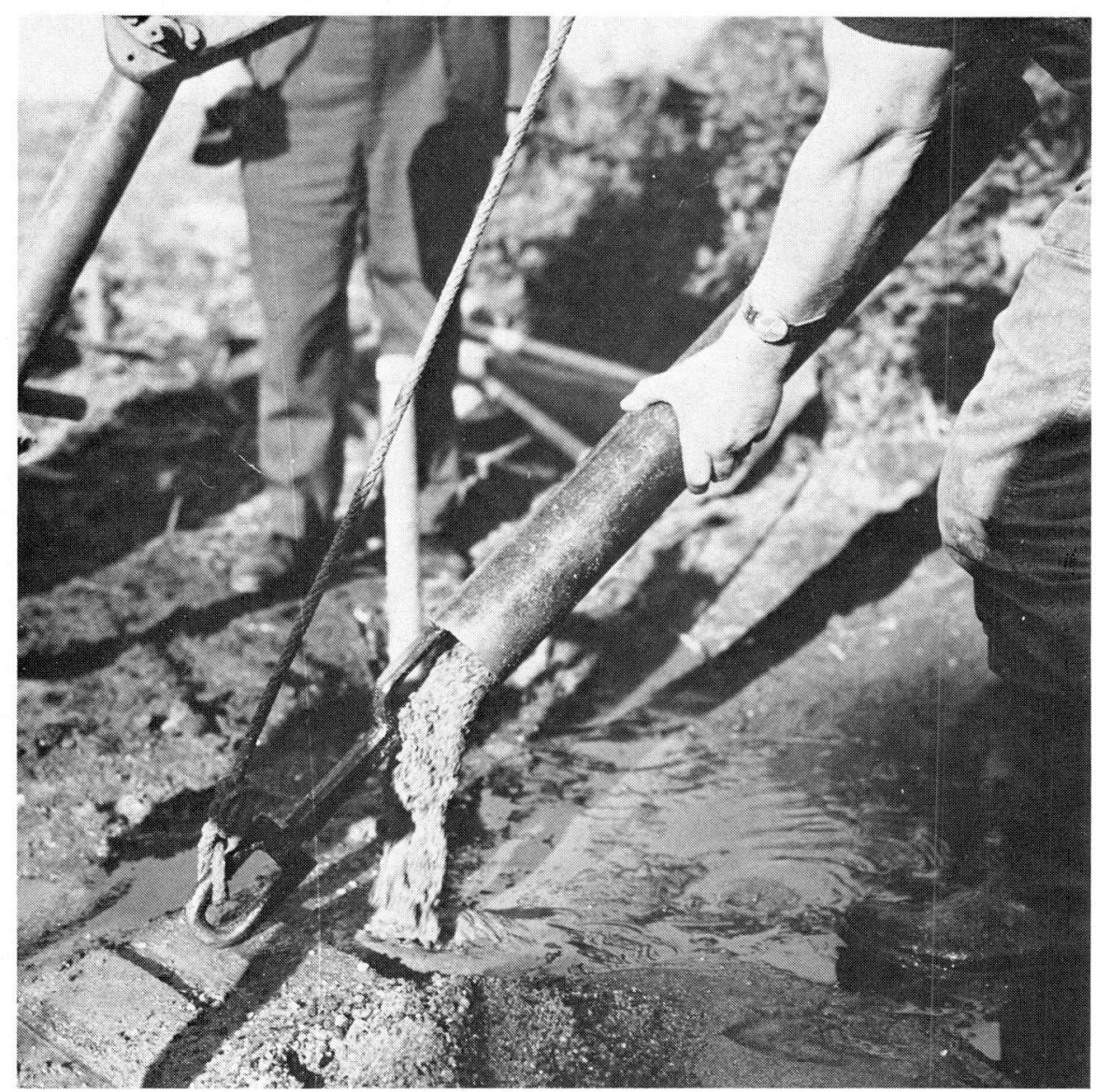

Abb. 7. Substrat-Ausschüttung aus Ventilbohrer. 

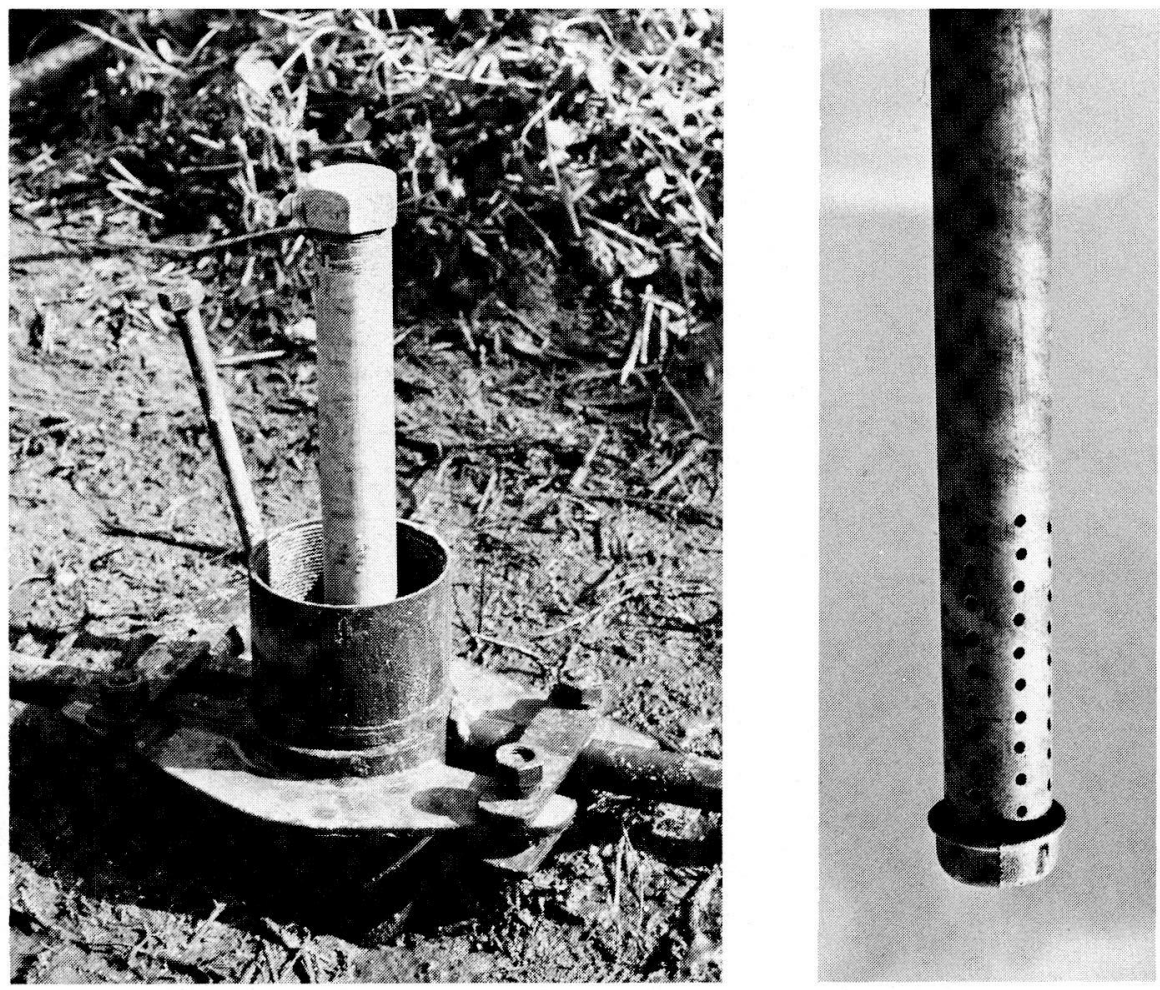

Abb. 8. a) Zentrisch in ein Bohrrohr eingesetztes Peilrohr;

b) unterer Peilrohrabschnitt mit Grundwassereinlauföffnungen $(\phi 5 \mathrm{~mm})$.

das gleichlaufende Auffüllen des Bohrloches. Hierzu wurden die vorher zutage geförderten Lockergesteine verwendet.

\section{b) Einrammen der Peilrohre mittels Presslufthammers}

Im Fuldatal wurden die mit Spitze versehenen Peilrohre durch die Bolzenschläge eines Presslufthammers in den Untergrund eingerammt (Abb. 9, 10). Zum Erreichen einer konstanten Treffsicherheit für den Schlagbolzen erhielt dieser eine sichere Führung in einem massiven Metalltrichter, der einer Verschlusskappe aufgeschweisst worden war, die jeweils dem einzurammenden Rohr aufgeschraubt wurde.

\section{Proben-Entnahme}

Die Entnahme von je einer Serie biologischer und chemischer Proben ist monatlich geplant; es wurde jedoch zunächst aus arbeitstechnischen Gründen mit Entnahmen in Januar, April, Juli, Oktober usw. begonnen. 


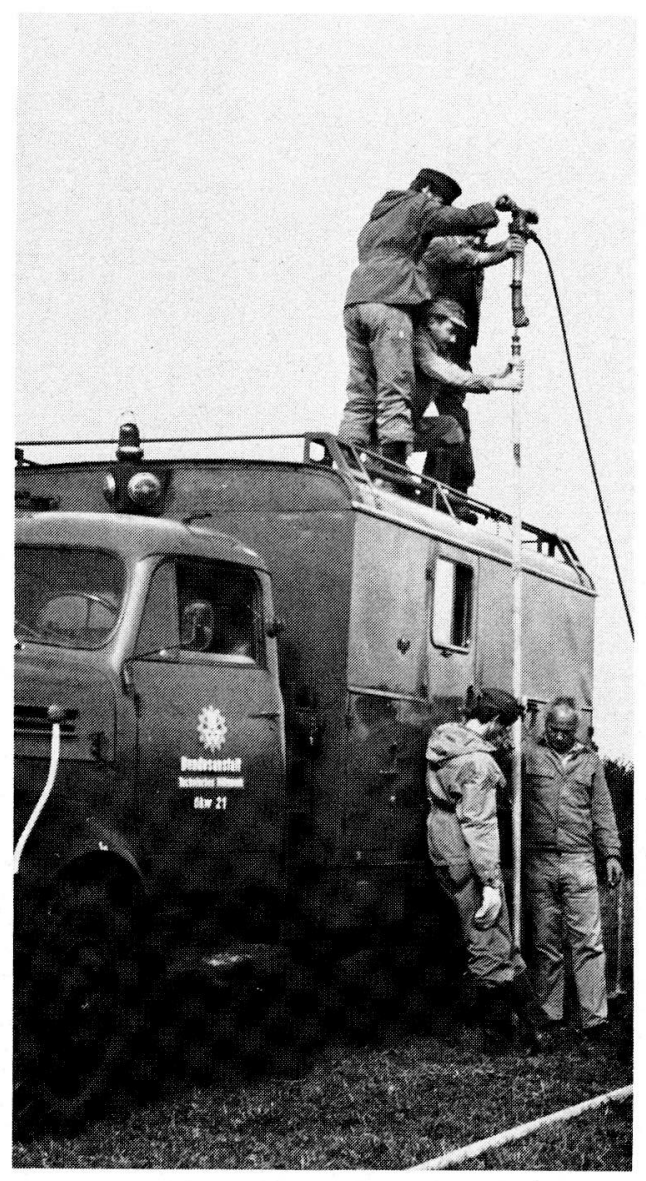

Abb. 9. Pressluft-Bohrung.

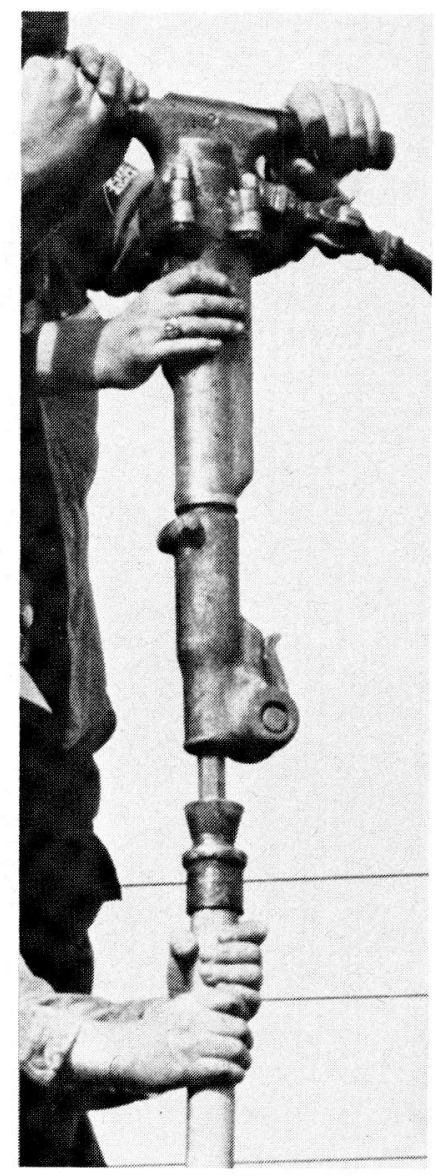

Abb. 10. Peilrohr mit Führungstrichter; Presslufthammer in Tätigkeit.

\section{a) Substrat-Proben}

Beim Bau der Weser-Station konnten auf Grund der bautechnischen Möglichkeiten (vgl. S. 278) Lockergesteins- Proben aus allen Tiefen entnommen werden, in denen die Einlauföffnungen der Peilrohre (Abb. 8, b) im anstehenden sandigkiesigen Grundwasserleiter stehen. Die Proben wurden beim Erreichen der betreffenden Bohrlochtiefen aus dem Ventilbohrer entnommen (Abb. 7). Ausserdem wurden nach gleichem Verfahren während des Bohrens laufend Substrat-Proben sichergestellt (alle $50 \mathrm{~cm}$ ), die dem Niedersächsischen Landesamt f. Bodenforschung, Hannover (Dr. Scherler) zur Ausführung von Substrat-Herkunfts-Untersuchungen zur Verfügung gestellt worden sind.

Die bei der Entnahme biologischer Proben (vgl. S. 287) zutage gepumpten Sand- 


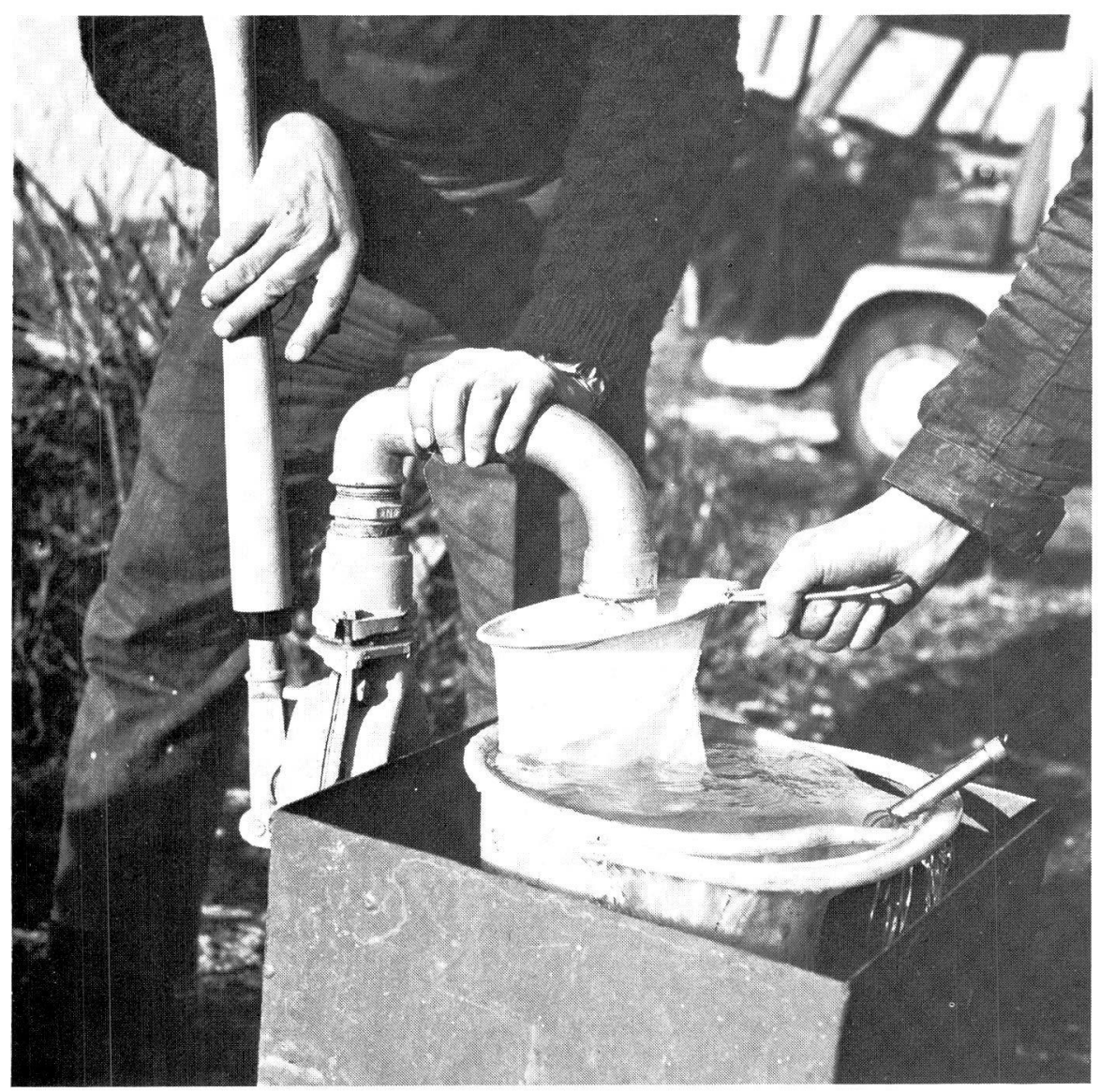

Abb. 11. Entnahme einer biologischen Probe aus einem Rohr der P.-Station Liebenau.

mengen wurden für granulometrische Untersuchungen in Plastik-Beuteln aufbewahrt, da ja mit einer fortlaufenden Ausschwemmung feinstkörniger Substratkomponenten zu rechnen ist: ein Vorgang der unter Umständen eine Erweiterung der subterranen Porensysteme und damit eine Biotopveränderung zur Folge haben könnte. Derartige Proben werden auch regelmässig aus der Station im Fuldatal entnommen.

\section{b) Wasserproben für biologische Untersuchung}

Eine auf das zu untersuchende Peilrohr aufgeschraubte Membranpumpe (Abb. 11) fördert jeweils 501 Grundwasser, das durch ein Beutelnetz aus Seidengaze (Maschenweite: $80 \mu$ ) hindurchgeleitet wird (Abb. 11). Die so heraufgepumpten 


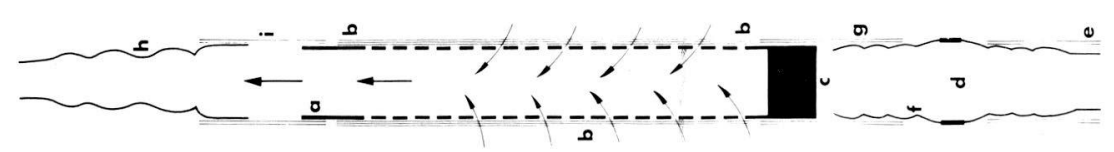

Abb. 12. Peilrohr-Filter. Erklärungen im Text.

Organismen verteilen sich dabei in einer stellenweise recht erheblichen Menge mitgeförderten Sandes (ca. $50-2000 \mathrm{~cm}^{3}$ ). Eine Trennung der Organismen und der dem Sande beigemengten pflanzlichen Zerfallsteile erfolgte nach einer an anderer Stelle schon ausführlich beschriebenen Ausschlämm-Methode (Husmann, 1958: 99). Das Gemenge von Detritus und Organismen wurde mit $40 \%$ igem Formol (1:10) konserviert.

\section{c) Wasserproben für chemische Untersuchungen}

Zur Entnahme von Grundwasserproben für chemische Analysen wurde eine Schlauchleitung (Silicon, innerer $\phi 10 \mathrm{~mm}$ ) in den Peilrohren bis in eine Tiefe von etwa $20 \mathrm{~cm}$ oberhalb der Grundwasser-Einlauföffnungen herabgelassen. Die Trübung des Fuldatal-Grundwassers machte es erforderlich, insbesondere für Sauerstoffbestimmungen schon im Peilrohr eine Filterung subaquatisch vorzunehmen. Hitrzu wurde ein Rohrfilter (Abb. 12) konstruiert. Dieser Filter besteht aus einem ca. $26 \mathrm{~cm}$ langen Stützrohr (Abb. 12,a) aus Polyäthylen (innerer $\phi 14 \mathrm{~mm}$, Wandung: $1 \mathrm{~mm}$ ). In etwa $3 \mathrm{~cm}$ Abstand sowohl vom Rohranfang als auch vom Rohrende ab verlaufen im Rohrmantel 5 Längsreihen von Perforationen ( $\phi$ der Löcher: $3 \mathrm{~mm}$, Abstände derselben voneinander: $6 \mathrm{~mm}$ ). Dieser Lochreihen-Abschnitt des Stützrohres wird mit chemisch reinem weissen Zellstoff umhüllt. Um während der Probennahme ein Ausfasern von Zellstoff zu vermeiden, wird der Zellstoff-Mantel durch Umwickeln mit Perlongaze verstärkt. Dieser Filtermantel aus Zellstoff und Gaze (Abb. 12,b) erhält festen Kontakt mit dem Filterrohr, indem er mit Hilfe einer dünnen Nylon-Schnur fest und unverschiebbar dem PerforationsAbschnitt des Rohres aufgepresst wird, und zwar durch enge Über-Kreuz-Wicklung. Das untere Ende des Stützrohres ist dicht von einem Gummistopfen (Abb. 12, c) verschlossen.

Um das Herablassen des Rohrfilters im Peilrohr zu erleichtern, wird mit dem durch Gummistopfen verschlossenen Rohranfang ein ca. $25 \mathrm{~cm}$ langer Metallstab ( $\phi$ $7 \mathrm{~mm}$ ) (Abb. 12, d) verbunden, der von einem Siliconschlauch (Abb. 12, e) wasserdicht umhüllt ist. Die Verbindung dieses Senkstabes mit dem Rohrfilter erfolgt durch ein Schlauchverbindungsstück (Abb. 12, f) dem ein kurzer Aufschiebeschlauch (Abb. 12, g) aufsitzt. Der Senkstab hat zudem die Aufgabe, ein Eintauchen des Rohrfilters in feinstkörnige, schlammige Sedimente zu verhindern, wie sie sich über dem unteren Peilrohrende absetzen können. Vor der Verwendung wird der Rohrfilter in destilliertes Wasser eingetaucht und dabei die Zellstoffhülle durch leichtes, pressendes Umfassen ausgequetscht*, um damit eine Bildung von störenden Luftbläschen zu vermeiden. Zur Entnahme werden Rohrfilter und Leitungsschlauch mittels eines Verbindungsstückes (Abb. 12, h) und einer Aufschiebemanchette aus Siliconschlauch (Abb.12, i) verbunden, und ausserdem der Arbeitsschlauch einer Schlauchpumpe (Abb. 13) an den Peilrohrschlauch angeschlossen.

\footnotetext{
* Bei mikrobiologischen Untersuchungen sterile Gummihandschuhe!
} 


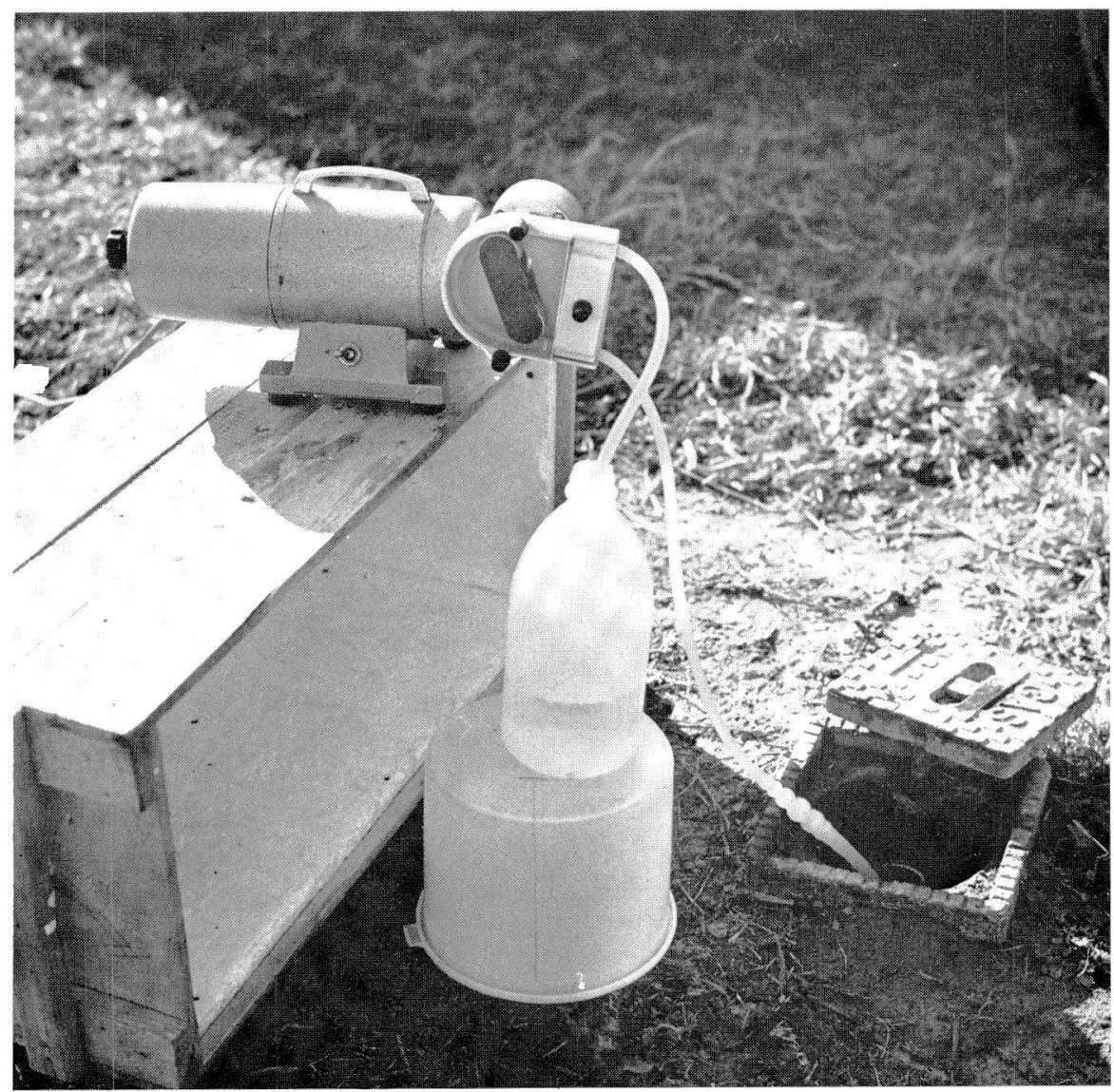

Abb. 13. Entnahme filtrierten Grundwassers aus einem unter Flur liegenden Rohr der Station Fulda-Aue.

\section{Untersuchungsmethoden}

Die Untersuchung der entnommenen Proben erfolgte auf folgende Weise:

\section{a) Granulometrische Substrat-Untersuchung}

Das getrocknete Substrat wurde mittels eines DIN-genormten Siebsatzes einer mit Elektromotor angetriebenen Prüfsiebmaschine (Modell "Lavib" ST 2/52, Siebtechnik G.m.b.H. Mühlheim/Ruhr) in Korngrössenfraktionen aufgeteilt; Angabe der Korngrössen-Anteile in Gewichtsprozenten. 


\section{b) Chemische Wasseruntersuchung}

pH-Wert: elektrometrisch: spez. Leitfähigkeit $\left(\mathrm{S} / \mathrm{cm}\right.$ bei $\left.20^{\circ} \mathrm{C}\right)$ : mit RadiometerGerät (Type LBR, Wiss. Techn. Werkstätten, Weilheim/Obb.); $\mathrm{KMnO}_{4}$-Verbrauch (PV): nach Deutsche Einheitsverfahren (DEV) H 4a; Sauerstoff: nach Winkler; P-Säureverbrauch (SV): nach DEV J 2 bzw. elektrometrisch titriert auf die entspr. pH-Werte; M-Säureverbrauch (SV): nach DEV J 2 bzw. elektrometrisch titriert auf die entspr. pH-Werte; Laugenverbrauch (LV): nach DEV H 7/8 bzw. elektrometrisch titriert auf die entspr. pH-Werte; Freie Kohlensäure $\left(\mathrm{CO}_{2}\right)$ : berechnet aus LV; Gesamthärte $\left({ }^{\circ} \mathrm{dH}\right)$ : komplexometrisch; Karbonathärte $\left({ }^{\mathrm{O}} \mathrm{dH}\right)$ : berechnet aus SV; $\mathrm{NH}_{4}$-N: nach F.A. Richards u. R.A. Kletsch, in: Strickland and Parsons (1965); $\mathrm{NO}_{2}-\mathrm{N}$ : mit Sulfanilamid (nach Bendschneider - Robinson (1952); $\mathrm{NO}_{3}-\mathrm{N}$ : nach Wood u.a. (1967); $\mathrm{PO}_{4}{ }^{3-}$ (gesamt) - P: nach Schmidt u. Ambühl (1965) m. Molybdänblau; Cl-: nach DEV (Chromat).

\section{c) Biologische Untersuchung}

Aussortierung der in Formol (4\%) konservierten Grundwassertiere unter Binokular-Lupe, Konservierung in 4\%igem Formol (Nematoda und Syncarida), Alkohol $(80 \%)$ - Nach 3stündiger Wässerung - (Copepoda u. Amphipoda), Bouin'scher Lösung (Troglochaetus), Lösung nach Navaschin (Oligochaeta).

\section{ERSTE ERGEBNISSE UND PROBLEME}

Da die ersten Befunde an unseren Peilrohrstationen bestimmte ökologische und hygienische Zusammenhänge bereits andeuten, soll nicht gezögert werden, darüber in gedrängter Form schon zu berichten, vor allem aber auch, um damit die Anregung zu geben, entsprechende Untersuchungen auch andernorts vorzunehmen, damit von möglichst vielen Seiten her die Notwendigkeit einer subterranen Umweltsicherung erkannt und bekanntgemacht werden kann.

\section{Das chemisch-biologische Vertikalprofil im Grundwasser der Weser-Niederterrase bei Liebenau}

In Hinblick auf die geographische Lage unserer Peilrohrstation Liebenau (Karte 2), konnte damit gerechnet werden, dass das dort erfasste Grundwasser unter Umständen von der Weser oder von der Aue her - Entfernung beider Flüsse jeweils ca. 2 $\mathrm{km}$ - beeinflusst wird, zumal der sandigkiesige Untergrund des Untersuchungsgebietes besonders durchlässig für strömendes Grundwasser ist. Daher wurden nicht nur aus dem Grundwasser Proben entnommen, sondern vergleichshalber auch aus Weser und Aue. Entnahmestellen: siehe Karte 2.

Ein Vergleich der Flusswasser- mit den Grundwasser- Untersuchungsergebnissen (Abb. 14) zeigt, dass Einflüsse von der Weser her sicher auszuschliessen sind; und wenn auch in der Weser und im grundwasserspiegelnahen Grundwasser annähernd gleiche Leitfähigkeitswerte festgestellt werden konnten (jeweils ca, $2600 \mathrm{~S} / \mathrm{cm}$ bei $20^{\circ} \mathrm{C}$ ), so kommt naturgemäss dem Chloridgehaltsverhältnis von Weserwasser und 


\begin{tabular}{|c|c|c|c|c|c|}
\hline & Aue & Weser & $\int_{2,25 m}^{X 11 d}$ & $\int_{2,75 \mathrm{~m}}^{\mathrm{X} 11 \mathrm{~d}}$ & $\int_{3,25 m}^{X I I d}$ \\
\hline pH-Wert & 7,17 & 7,63 & 6,20 & 6,19 & 6,67 \\
\hline $\begin{array}{l}\text { spez. Leitfähigkeit } \\
\mu \mathrm{S} / \mathrm{cm} \text { bei } 20^{\circ} \mathrm{C}\end{array}$ & 498 & 2600 & 2610 & 1440 & 1440 \\
\hline Gesamthärte ${ }^{\circ} \mathrm{dH}$ & 10,00 & 27,25 & 57,00 & 38,85 & 30,50 \\
\hline Carbonathärte ${ }^{\circ} \mathrm{dH}$ & 3,8 & 7,4 & 8,9 & 4,15 & 19,4 \\
\hline $\mathbf{M g}^{2+} \mathrm{mg} / \mathrm{l}$ & 5,6 & 65,4 & 17,2 & 34,8 & 40,7 \\
\hline $\mathrm{Cl}^{-} \mathrm{mg} / \mathrm{l}$ & 67,4 & 900,0 & 99,5 & 60,2 & 163,0 \\
\hline $\mathrm{SO}_{4}{ }^{2-}$ & 83,8 & 155,0 & 1262,0 & 510,0 & 183,0 \\
\hline
\end{tabular}

Abb. 14. Chemismus von Oberflächenwasser und Grundwasser bei Station Liebenau XIId.

XII $d$ - Grundwasser eine hier allein entscheidende Aussagekraft zu. Denn ganz offensichtlich wirkt sich die starke "Versalzung" der Weser $\left(\mathrm{Cl}^{-}: 900 \mathrm{mg} / 1\right)$ nicht im untersuchten Grundwasser aus $(99,5 \mathrm{mg} / 1)$; und gerade dieses nahe bei 10: 1 liegende Chloridmengenverhältnis spricht eindeutig dafür, dass Einflüsse von der Weser her, wie schon gesagt, sicher nicht in Betracht gezogen werden können, weil ja die Menge der im Wasser gelösten Chloride bekanntermassen nicht durch intergranulare biologische Selbstreinigungseffekte während der Lückensystempassage vermindert werden kann!

Eine recht geringe Grundwasserbeeinflussung von der Aue her ist auf Grund der Befunde nicht auszuschliessen; ein überzeugender Nachweis aber lässt sich an Hand der vorliegenden Daten nicht erbringen. Die durch Schlagen von Einzelpeilrohren vorgesehene Feststellung der Grundwasser-Strömungsrichtung wird hierzu sicher weitere Aufschlüsse geben.

Bei den Grundwasseruntersuchungen von der Peilrohrstation XIId aus kann also davon ausgegangen werden, dass die Beschaffenheit des dort erfassten Grundwassers, wenn überhaupt, dann aber sicher nur geringfügig von der Aue her fluviatil beeinflusst werden könnte! Und daher sind die von der Station aus festzustellenden Verunreinigungs-Indikatoren auf Versickerungen zurückzuführen, die zumindest im wesentlichen von Müllablagerungen der nächsten Umgebung ausgehen (vgl. S. 273) und ferner unter Umständen auch von gedüngten Feldern und Wiesen des weiteren Umlandes; letzteres in Anteilen, die voraussichtlich ebenfalls mittels der oben erwähnten Schlagung von Einzel-Peilrohren ermittelt werden könnten.

Doch nun zu den ersten Befunden! Am Untersuchungstage (25. Januar 1971) lag der Grundwasserspiegel $1.70 \mathrm{~m}$ unter der Erdoberfläche. Die wasserchemischen Einflüsse der im Untersuchungsbereich lagernden Unratsstoffe wirkten sich bis in 

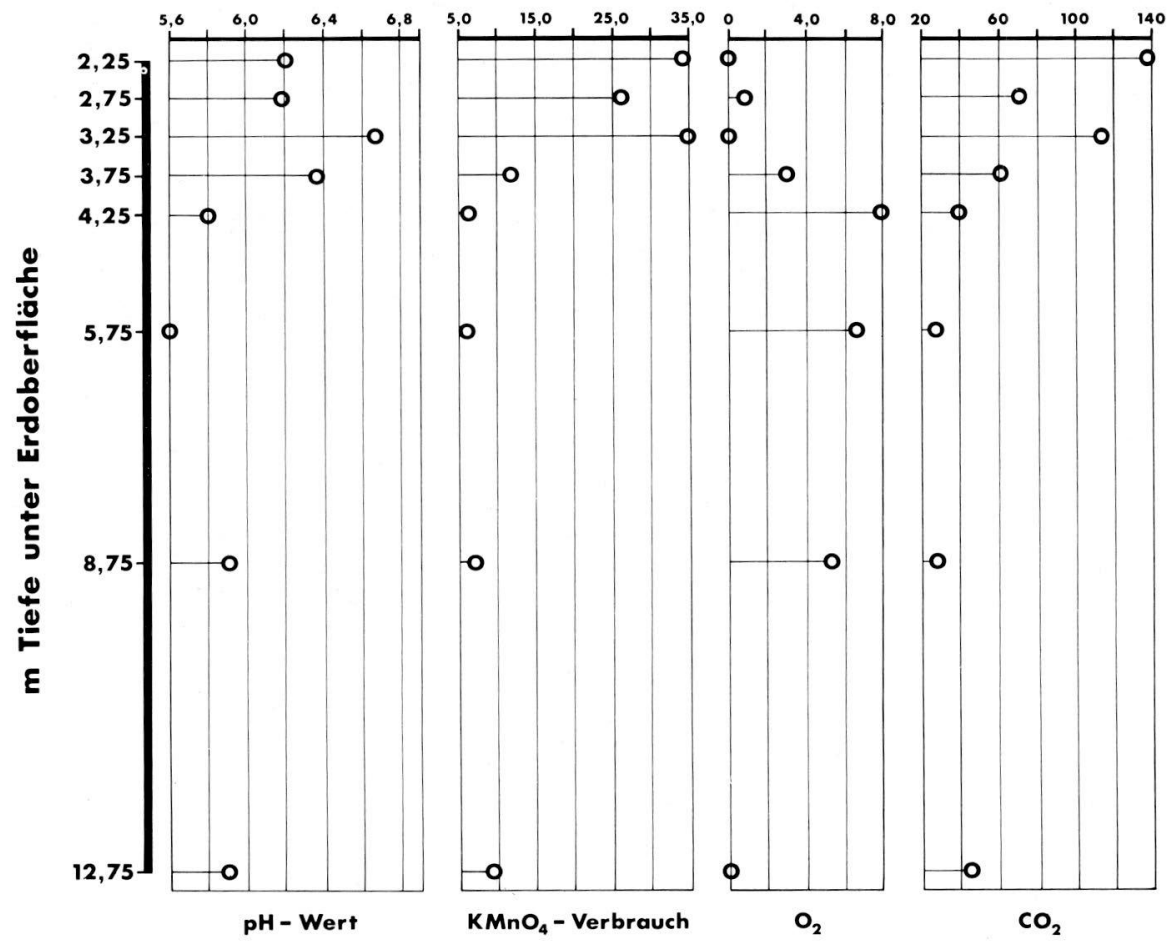

Abb. 15. Die chemische Grundwasser-Beschaffenheit im Vertikal-Profil XIId bei Liebenau (a). Mengen-Angaben: mg/1

eine Tiefe von ca. $3.50 \mathrm{~m}$ unterhalb der Erdoberfläche aus: das Grundwasser wurde also bis hinab in eine Tiefenlage von ca. $1.80 \mathrm{~m}$ unterhalb des Grundwasserspiegels erheblich verunreinigt (vgl. Abb. 14, 15, 16, 17, 18).

Diese den Grundwasserspiegel unterlagernde Verunreinigungszone führt Interstitialwasser, das in Lückensystemen von Substratkomponenten besonders geringer Korngrösse (vgl. Abb. 2) nur überaus langsam zieht. Diese geringe Strömungsgeschwindigkeit in den korngrössenbedingt hier nur recht englumigen Sandlückensystemen gewährt den gelösten infiltrierenden Substanzen naturgemäss nur einen entsprechend geringen Verdünnungsgrad (vgl. A. Ruttner-Kolisko, 1962) und führt daher zwangsläufig zur Herausbildung einer besonders deutlich ausgeprägten grundwasserspiegelnahen ökologischen Verödungszone, deren erheblich gestörter biologischer und chemischer Zustand (Abb. 14, 15, 16, 17, 18) bei Anreicherung mit Indikatoren für Wasserverschmutzung vor allem durch Sauerstoffschwund und Faunenleere gekennzeichnet ist!

$\mathrm{Zu}$ grösserer Tiefe hin verlieren sich alle den Gütegrad des Interstitialwassers beeinträchtigenden gelösten chemischen Substanzen (Abb. 15, 16, 17, 18). Das Intergranularwasser aus $3.75,4.25,5.75 \mathrm{~m}$ Tiefe unter der Erdoberfläche führt 
sogar bis zu 7,8 mg/1 Sauerstoff und wird von einer Lebensgemeinschaft stygobionter Grundwassertiere besiedelt (vgl. Husmann, 1968a u. Abb. 18). Zu noch grösserer Tiefe hin ist dieser Biocoenose wiederum eine Grenze gesetzt: in $12.75 \mathrm{~m}$ Tiefe herrscht bei völligem Sauerstoffschwund absolute Faunenleere (Abb. 15 u. 18). Hier wird offensichtlich die von oben her durch naturwidrige Einflüsse eingeengte subterranaquatile Metazoen-Biocoenose von der Tiefe her durch Grundwasser begrenzt, das von Natur aus sauerstoffrei ist!

\section{Das chemisch-biologische Vertikalprofil im Grundwasser der Fulda-Aue bei Fulda}

Das aus der Peilrohr-Station Fulda-Aue/26 - Entfernung vom Fluss ca. $500 \mathrm{~m}$ geförderte Grundwasser deutet nach bisher vorliegenden ersten Befunden nicht überzeugend auf subterrane, vom Fuldabett ausgehende Infiltrationen hin (Flusswasser-Entnahmestelle: Karte 3). Auch im Fuldatal kann nur mittels einer Peilrohrreihe (Vgl. S. 278), die vom Flussufer bis zur Station verlaufen wird, versucht werden, eindeutige Aufschlüsse über fluviatile Infiltrationsvorgänge zu erlangen.

Offensichtlich - das wurde bereits vermerkt - ziehen andererseits von den Talhängen (Karte 3) aus Buntsandstein, Muschelkalk, Keuper und Diluvium subterrane Wässer in den untersuchten Talauen-Grundwasserstrom; und ohne Zweifel
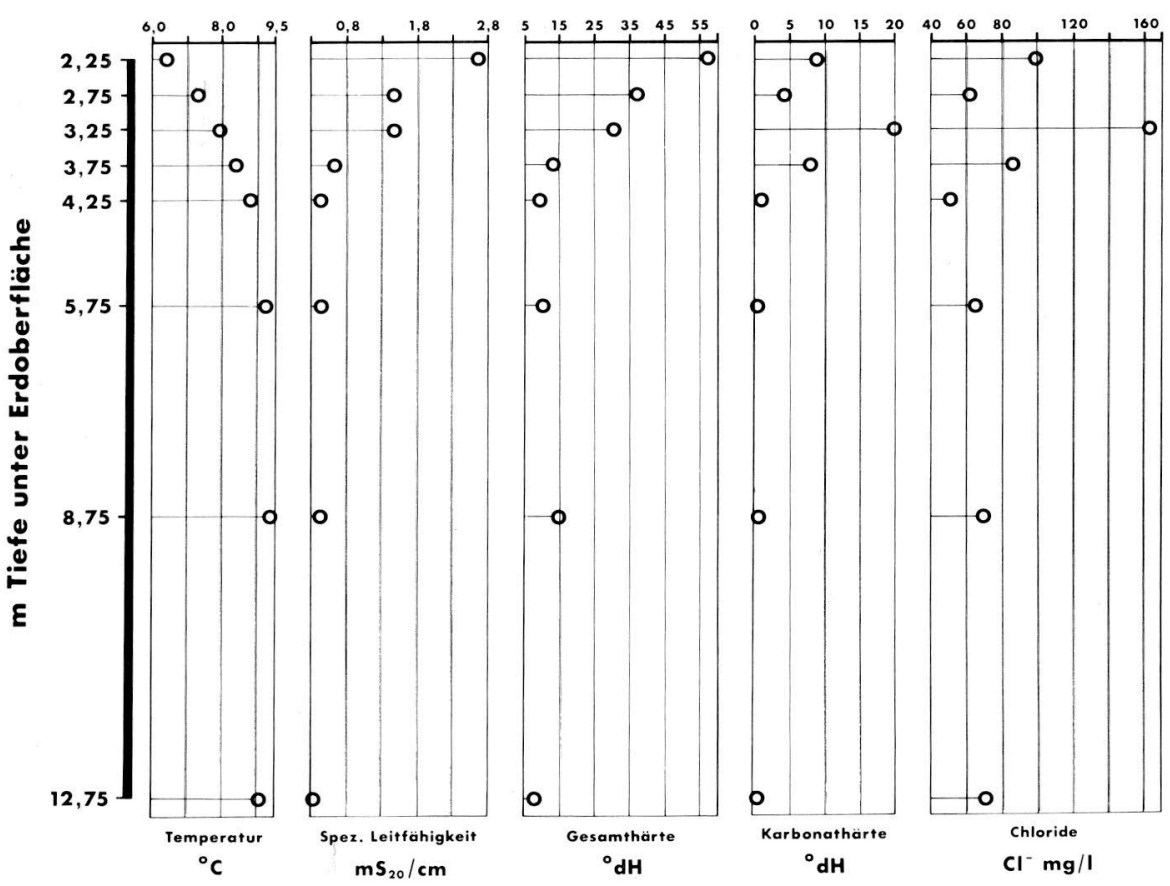

Abb. 16. Die chemische Grundwasser-Beschaffenheit im Vertikal-Profil XIId bei Liebenau (b). 
beeinflussen diese zum Untergrund der Fulda-Aue hin zutal strömenden Grundwässer erheblich den Chemismus des subterranen Talauenwassers, wobei offenbar gedüngte Wiesen und Felder als Infiltrations-Areale angesehen werden müssen!

Geringer Gehalt an Sauerstoff, völliges Fehlen von Grundwassertieren (Abb. 20, 21) sowie gelöste Verunreinigungsanzeiger - Chloride, Ammoniak, Nitrate (Abb. 19) - kennzeichnen dieses grundwasserspiegelnahe, epistygale Talauengrundwasser ganz besonders. Eine nur $50 \mathrm{~cm}$ tiefer - aus $3 \mathrm{~m}$ unterhalb der Erdoberfläche entnommene Probe zeigt, dass zur Tiefe hin geradezu schlagartig ökologisch hochgradig andersartige wasserchemische und biologische Gegebenheiten herrschen: beispielsweise eine Verdreifachung der Sauerstoffmenge, ausserdem anstelle der im Epistygal herrschenden Faunenleere: dichte Besiedlung durch Grundwassertiere (Abb. 21, 22). Nur weitere $50 \mathrm{~cm}$ tiefer zeigt sich wiederum eine sprunghafte Veränderung in Faunenbild und Wasserchemismus: Rückgang des Sauerstoffs auf ein Drittel, Verringerung der Biocoenosendichte auf ungefähr ein Hundertstel. Noch weitere $50 \mathrm{~cm}$ tiefer: erneuter Anstieg der im Lückenwasser gelösten Sauerstoffmenge um ein Drittel, Zunahme der Individuendichte innerhalb der Grundwasser-Lebensgemeinschaft um etwa das 30 fache.

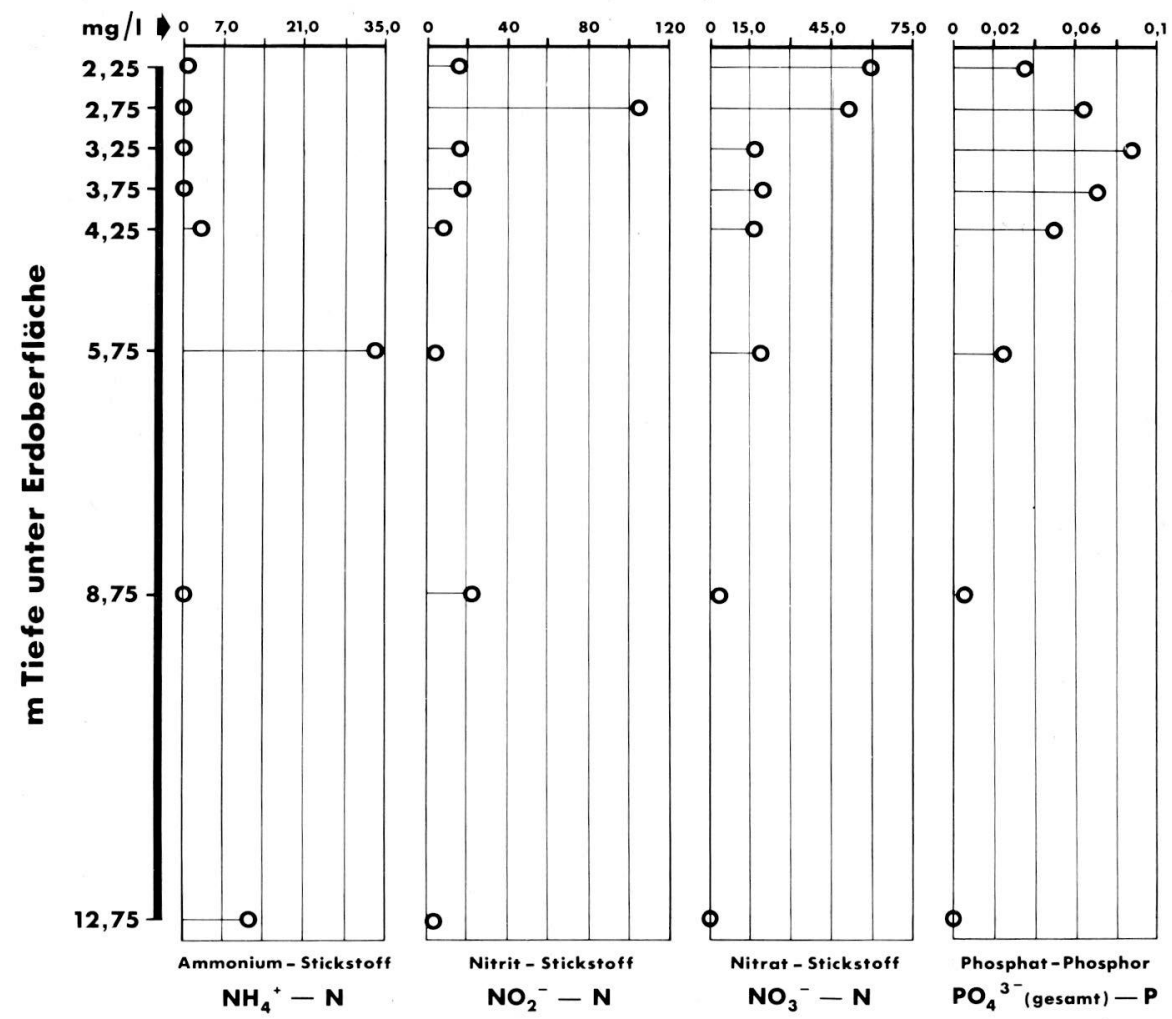

Abb. 17. Die chemische Grundwasser-Beschaffenheit im Vertikal-Profil XIId bei Liebenau (c). 


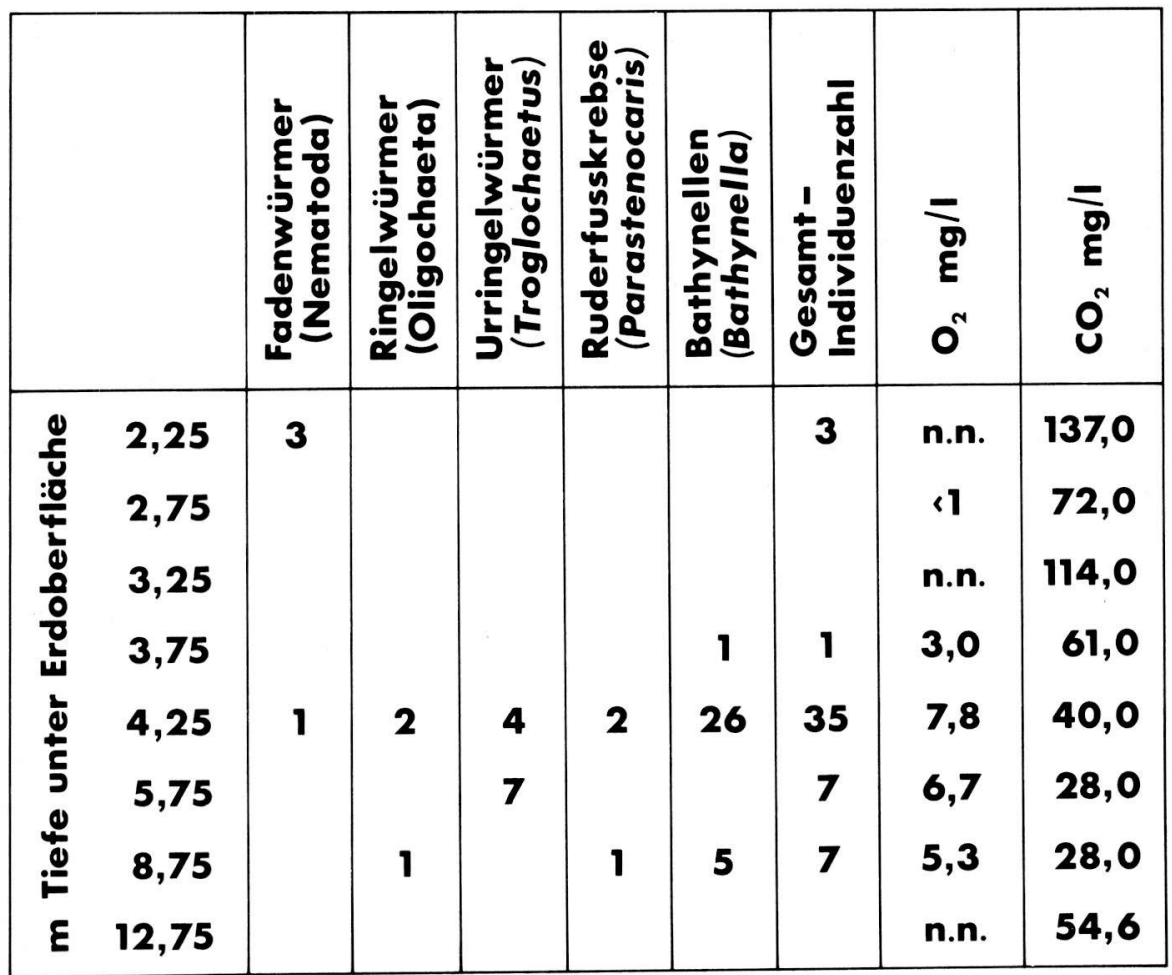

Abb. 18. Die vertikale Verteilung von Grundwassertieren in der Niederterrasse der Unterweser bei Liebenau (P.-Station XIId) und ihre Abhängigkeit vom Sauerstoffgehalt des Interstitialwassers,

\section{DIE ÖKOLOGISCH-HYGIENISCHE AUSSAGE DER BEFUNDE}

Die ersten Befunde über die vertikale Verteilung von Organismen und chemischen Substanzen im Grundwasser sandigkiesiger Lockergesteine dürfen sicher nur als ökologische Momentaufnahmen einer im Jahreslauf mehr oder weniger wandelbar verlaufenden chemisch-biologischen Zustandsfolge gewertet werden. Es würde daher sicher verfrüht sein, aus diesen Ergebnissen ins einzelne gehende Schlussfolgerungen ziehen zu wollen!

Ohne Zweifel werden aber mit diesen Anfangsbefunden sowohl grundwasserökologische Naturgegebenheiten als auch deren Störungen sichtbar gemacht, die infolge von Schadstoff-Infiltrationen ausgelöst werden können.

Als besonders auffallende stygolimnologische Naturgegebenheit ist offenbar die bisher meines Wissens noch nicht aus Talauen und diluvialen Terrassen bekannte relativ dichte Folge verschiedenartig beschaffener Grundwasserkörper zu vermer- 

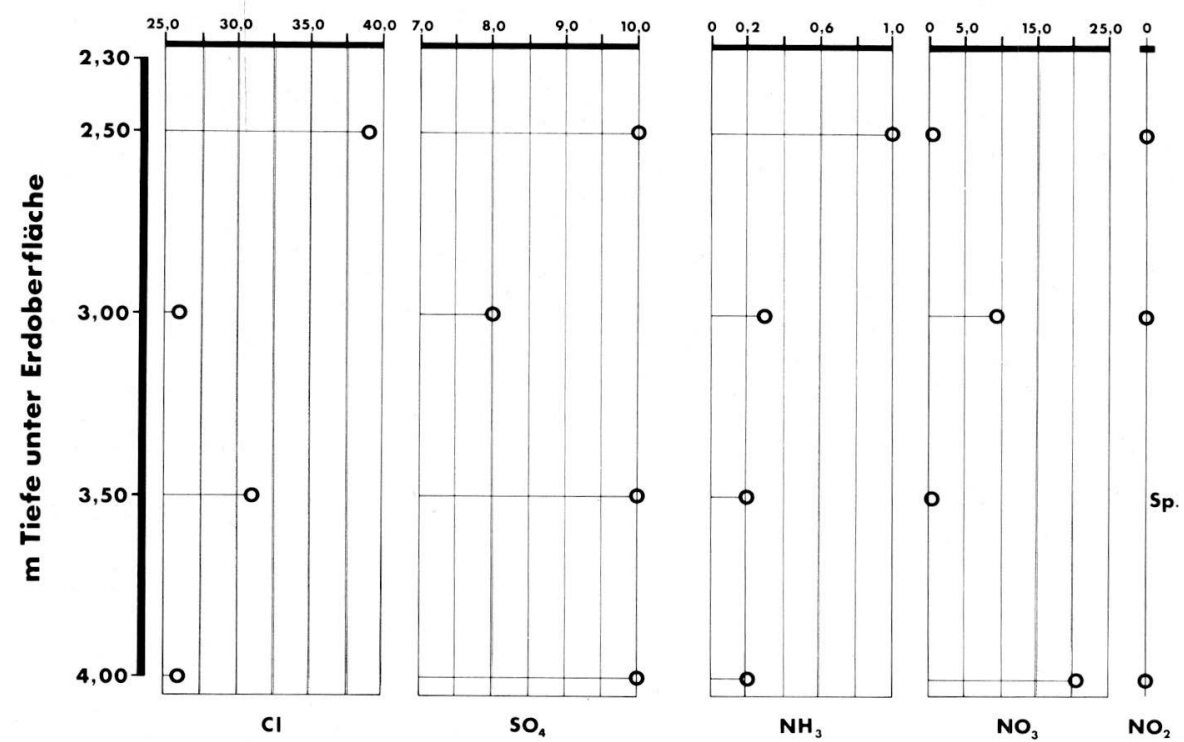

Ab.. 19. Die chemische Grundwasser-Beschaffenheit im Vertikal-Profil der Fulda-Aue (a). Mengen-Angaben:mg/1

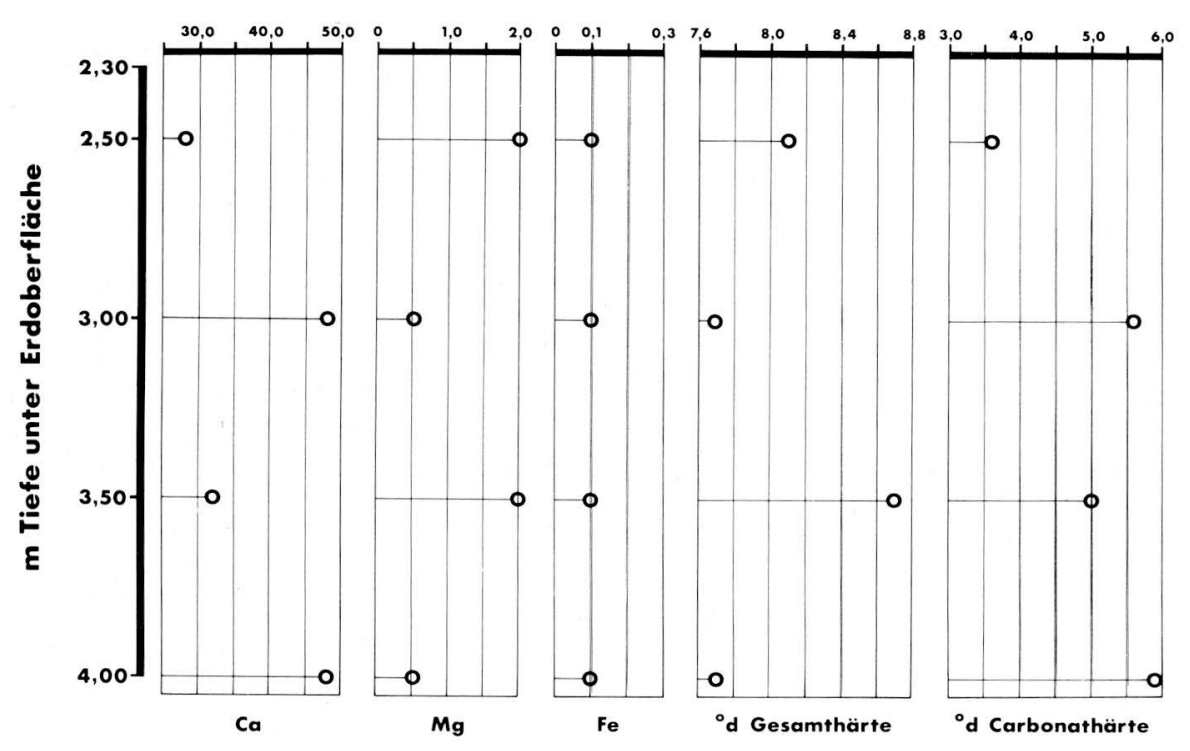

Abb. 20. Die chemische Grundwasserbeschaffenheit im Vertikal-Profil der Fulda-Aue (b). Mengen-Angaben: $\mathrm{mg} / 1$ 
ken, bei denen es sich aber sicher nicht um flächenartig ausgedehnte Grundwasserschichten handeln dürfte, sondern um subterrane Wässer in Teilbereichen eines Raummosaiks verschiedenartiger Lockergesteinsablagerungen, deren Schräg- und Kreuzschichtung (vgl. Brinkmann 1950, Abb. 16) und Einlagerung von Sand- und Tonlinsen ein wechselvolles Nebeneinander und Untereinander stygoökologischer Verhältnisse geben kann. Dieses Phänomen einer grundwasserspiegelnah überaus dichten Folge verschiedenartig beschaffener Grundwässer konnte bei GrundwasserUntersuchungen im südlichen Wiener Becken (Liepold 1960) noch nicht ermittelt werden, weil hier im Verlauf von Tiefbohrungen nur im Abstand von durchweg 10 und mehr Metern Vertikalproben entnommen wurden, die dann ebenfalls stark differenzierte Grundwässer bis aus $150 \mathrm{~m}$ Tiefe zutage förderten.

Das bei Liebenau und Fulda festgestellte ausserordentlich dichte intergranulare Übereinander sehr differenzierter Grundgewässer ist in der Fulda-Aue besonders ausgeprägt: zwischen den Tiefenlagen 250 und $400 \mathrm{~cm}$ unterhalb der Erdoberfläche konnten 4 chemisch und biologisch durchaus verschiedenartige Subterrangewässer nachgewiesen werden (vgl. S. 294 u. Abb, 19, 20, 21, 22). Mit anderen Worten: alle 4 zur Tiefe hin nur jeweils $50 \mathrm{~cm}$ untereinanderliegenden Entnahmestellen lieferten sehr unterschiedliches Grundwasser.

Aber auch die vertikale Folge der Grundwässer von Liebenau ist sozusagen durch eine Stapelung verschieden beschaffener Grundwässer gekennzeichnet (vgl. Abb. 15, $16,17,18)$.

Das stärkere Ausgeprägtsein dieses Schichtungs-Phänomens im Fuldatal ist offenbar geomorphologisch bedingt. Denn die geologisch abwechslungsreichen hügeligen Talhänge (Karte 3) führen dem Untergrund der Fulda-Aue verschiedenartiger

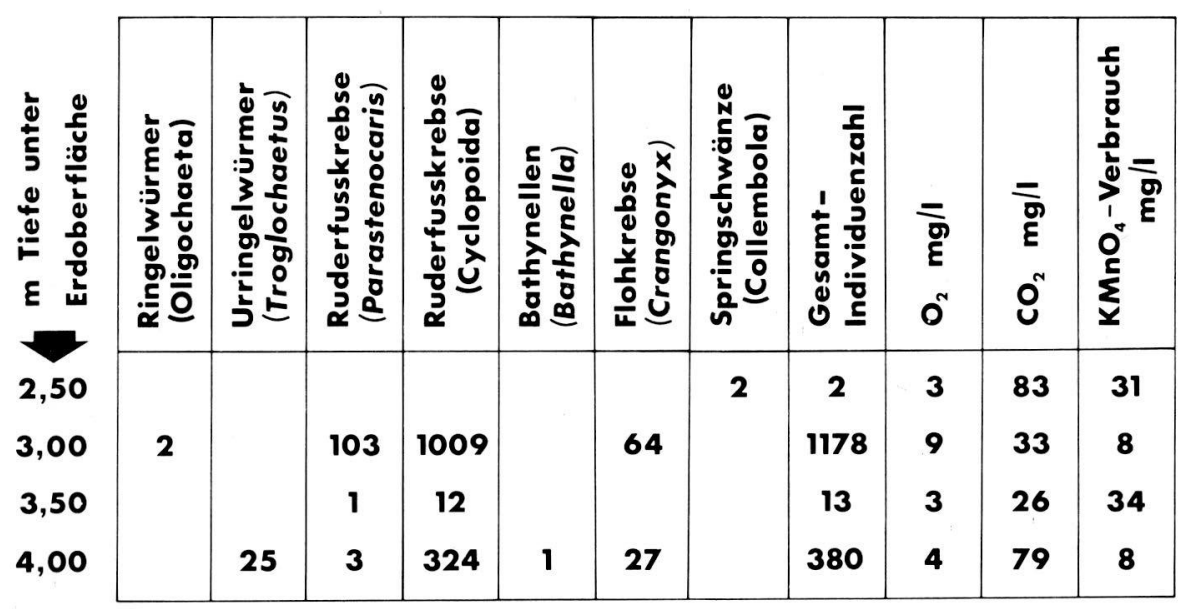

Abb. 21. Die vertikale Verteilung von chemischen Substanzen und Grundwassertieren in der Fulda-Aue (P.-St. 26). 
beschaffene Grundwässer zu, als es vergleichsweise in der Fast-Ebene bei Liebenau geschehen kann.

Von besonders ausschlaggebender Bedeutung für die erwähnte Differenziertheit der aus den verschiedenen Tiefen zutage geförderten Grundwässer dürfte zweifellos die Korngrössenverteilung der jeweils durchströmten Lockergesteinsablagerungen sein, durch die wiederum Hohlraumvolumen und Strömungsgeschwindigkeit bedingt werden und damit die Sicherstellung der Zufuhr von Sauerstoff und Grundnahrungsstoffen in Gestalt organismischer Zerfallsteile für sich entfaltende Biocoenosen von Grundwassertieren, so wie das in den subterranen Biozonen unserer Peilrohrstationen der Fall ist!

Die oben erwähnten Störungen der subterranökologischen Gegebenheiten durch infiltrierende Schadstoffe führen ebenfalls in beiden Untersuchungsstellen zu im Prinzip gleichen Phänomenen, nämlich zur Herausbildung einer Mineralisationszone unterhalb des Grundwasserspiegels, in der es zu einer mehr oder weniger spontanen Abnahme von Verunreinigungstoffen - zu biologischen Reinigungseffekten! kommt, allerdings nur solange, bis die biocoenotische Abbaufähigkeit überfordert wird, wie in der untersuchten Niederterrasse bei Liebenau und in der Fulda-Aue, wo ja eine Zone ökologischer Verödung bereits in die tieferliegende sauerstoffreiche Biozone vordringt; ein Warnzeichen, das die Gefahr deutlich erkennen lässt, in der sich grundwasserführende Sande und Kiese - natürliche Speicher guten Trinkwassers! - vielerorts bereits befinden!
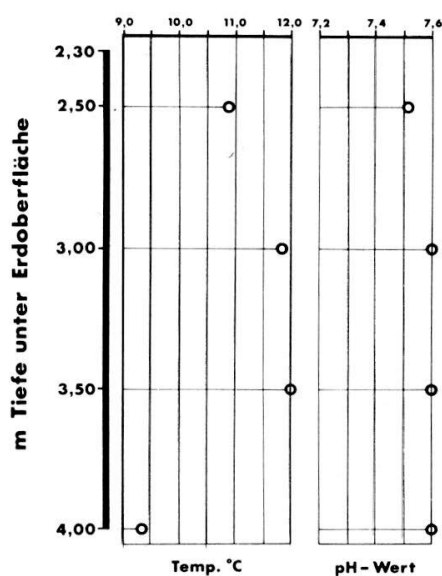

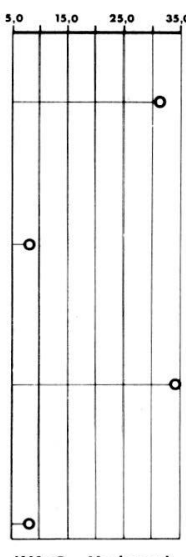

KMnO, - Verbrauch

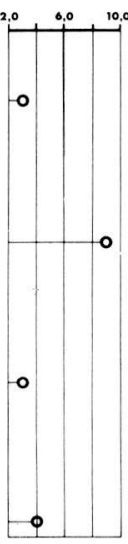

$\mathbf{O}_{2}$

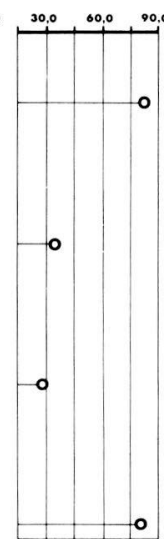

$\mathrm{CO}_{2}$

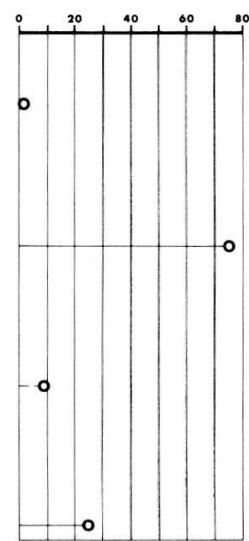

$\%$ Ges. - Individuenzahl (1573)

Abb. 22. Die vertikale Verteilung von Grundwassertieren in der Fulda-Aue (P.-St. 26) und ihre Abhängigkeit vom Sauerstoffgehalt des Interstitialwassers.

Mengen-Angaben: $\mathrm{mg} / \mathrm{l}$ 


\section{DANK UND HINWEISE}

Den Harzwasserwerken des Landes Niedersachsen und der Stadtwerke Fulda G.m.b.H. habe ich für vielseitige Hilfeleistungen zu danken: Technische Beratungen, Herstellung der Peilrohre, Ausführung der Tiefbau-Arbeiten, Stellung von Hilfskräften bei der Entnahme von Wasserproben, Durchführung chemischer Wasseranalysen. Insbesondere danke ich Herrn F. Keipert, Stadtwerke Fulda, sowie Herrn Dipl. Chem. G. Matzke nebst Mitarbeitern vom Zentrallabor der Harzwasserwerke für die Ausführung der chemischen Wasseranalysen. Herrn Dr. J. Brehm, Limnologische Fluss-Station, Schlitz gebührt bester Dank für wertvolle Anregungen bei der Beurteilung chemischer Befunde. Mit Dank besonders erwähnt zu werden verdient auch das Einsatzkommando der Bundesanstalt Technisches Hilfswerk, Fulda, für das mühevolle Einrammen der Peilrohre in den Untergrund der Fulda-Aue.

Allen Helfern an beiden Stationen sei inbesondere herzlich gedankt für die immer gute Zusammenarbeit!

Die Karten $1-3$ sowie die graphisch gestalteten Abbildungen wurden nach Entwürfen des Verfassers angefertigt: Uta Schnell, Limnologische Fluss-Station, Schlitz.

\section{ZUSAMMENFASSUNG}

Aus Lockergesteins-Untergrund der Fulda-Talaue und einer Niederterrasse der Unterweser wird Grundwasser aus verschiedenen Tiefenlagen (Abb. 1-4) für chemische und biologische Untersuchungen zutage gefördert. Hierzu wurden an den Untersuchungsstellen Peilrohr-Anlagen eingerichtet.

Der Bau der Peilrohrstationen erfolgte mittels Ventilbohrer- und Presslufthammer-Methode (Abb. 5-10). Diese Verfahren und die Methoden zur Probennahme und - untersuchung werden in gedrängter Form dargestellt.

An beiden Untersuchungsstellen werden insbesondere die Einwirkungen der vertikalen Infiltration von Verunreinigungen auf Wasserbeschaffenheit und Grundwasserorganismen untersucht. Hierzu ist die Niederterrassen-Station besonders aussagekräftig, da sie in einer durch Müll und Fäkalienschlamm erheblich verunstalteten Sandgrube liegt.

Die ersten Befunde (Abb. 15 - 22) zeigen, dass die zum Abbau der infiltrierenden Schadstoffe führenden mikrobiologisch-chemischen Vorgänge besonders intensiv dicht unter dem Grundwasserspiegel verlaufen: in einer "intergranularen Sprungschicht" (Husmann 1971a). Diese besonders aktive Mineralisationszone erwies sich als sauerstoffarm bis sauerstoffrei und nicht von Grundwassertieren besiedelt.

Die biologischen Reinigungseffekte der epistygal-intergranularen Mineralisationszone können entarten, wenn die vertikale Zufuhr von Verunreinigungstoffen die biocoenotische Mineralisationspotenz überfordert. Wie unsere Befunde - geradezu als Warnzeichen! - zeigen, wird dann die intergranulare Sprungschicht zu einer vertikal nach unten vordringenden epistygalen Verödungszone.

Die in unterirdischen Gewässern durch Verunreinigungen hervorgerufene Störung 
des biologischen Gleichgewichtes kann sich als Beeinträchtigung der Trinkwassergüte auswirken, sofern die betreffenden Wässer der menschlichen Versorgung dienen.

Daher werden bei der Fortsetzung der Peilrohr-Untersuchungen Probleme der Grundlagenforschung, der Trinkwasserhygiene und des subterranaquatilen Lebensraumschutzes als engzusammengehörige Fragestellungen behandelt werden.

\section{SUMMARY}

For chemical and biological investigations groundwater was brought to light from different depths of the sandy and gravelly underground of the Fulda river as well as from the subterranean region of a diluvial terrace of the Weser (fig. 1-4). For this purpose pipe-stations were installed by using either a valve borer or a pneumatic hammer (fig. $5-10$ ).

A concise description of the methods of drilling, taking groundwater and subterranean organisms, and the analysis of this material was given.

In both stations the influences were considered, which vertical infiltrations of polluted water have on the quality of groundwater and the living-conditions of its inhabitants. In this respect the station in the Weser valley is most instructive, since it is located in a sand-pit used as a dust-hole.

First results (fig. $15-22$ ) demonstrate that microbiological activities and chemical processes connected with the decomposition of organic matter are most intensive in a zone just below the groundwater-surface, called "intergranulare Sprungschicht" (Husmann 1971a).

In this zone there is very little oxygen or even none; so groundwater organisms are lacking here.

If, however, the water pollution overtaxes the mineralisation-capacity of the biocoenosis, the biological filtering effects will be reduced markedly. Then the "intergranulare Sprungschicht" will degenerate to a zone of desolation extending into deeper layers; in this sense our results can be understood as a warning.

The disturbance of the biological equilibrium in the intergranular groundwater caused by pollution can reduce its value for drinking-water purposes.

In future investigations on this subject problems of basic research, hygiene of drinking-water and the preservation of aquatic subterranean biotops will be treated as one.

\section{LITERATUR}

AMBÜHL, H. \& SCHMID, M. (1965): Die Bestimmung geringster Mengen von Phosphation im Wasser von Binnenseen. - (Beiträge zur chemischen Wasser- und Abwasseranalyse, Nr. 1). - Schweiz. Zeitschr. f. Hydrol. 27: 172-183.

AMBÜHL, H. \& SCHMID, M. (1965a): Die Bestimmung deringster Mengen von Gesamtphophor im Wasser von Binnenseen. (Beiträge zur chemischen Wasser- und Abwasseranalyse, Nr. 2) - Schweiz. Zeitschr. f. Hydrol. 27: 184-192. 
BRINKMANN, E. (1950): E. KAYSER'S Abriss der Geologie. - F. Enke Verlag, Stuttgart, 296 $\mathrm{S}$.

DAMRATH, H. (1963): Dahlhaus-Damrath, Wasserversorgung. - In: Teubners Fachbücher f. Hoch. - u. Tiefbau, Stuttgart, $177 \mathrm{~S}$.

FARKASDI, G., GOLWER, A., KNOLL, K. H., MATTHESS, G. \& SCHNEIDER, W. (1969): Mikrobiologische und hygienische Untersuchungen von Grundwasserverunreinigungen im Unterstrom von Abfallplätzen. - Städtehygiene 2: 23 - 31.

GOLWER, A., MATHESS, G. \& SCHNEIDER, E. (1969): Selbstreinigungsvorgänge im aeroben und anaeroben Grundwasserbereich. - Vom Wasser 36: 64 - 92.

GOLWER, A., KNOLL, K. H., MATTHESS, G., SCHNEIDER, W. \& WALLHAUSER, K. H. (1972): Mikroorganismen im Unterstrom eines Abfallplatzes. - Gesundheits-Ingenieur 5: 142-152.

HUSMANN, S. (1958): Untersuchungen über die Sandlückenfauna der bremischen Langsamfilter. - Abh. Braunschw. Wiss. Ges., 10: $93-116$.

(1961): Filtersandschichten in Grundwasser-Anreicherungsanlagen als künstliche Biotope aquatiler Subterranorganismen. - Abh. Braunschw. Wiss. Ges. 13: 163-181.

(1962): Ökologische und verbreitungsgeschichtliche Studien über den Archianneliden Troglochaetus beranecki Delachaux; Mitteilung über Neufunde aus den Grundwasserströmen von Donau, Ybbs, Ötz, Isar, Lahn, Ruhr, Niederrhein und Unterweser. Zool. Anz. 168: 312-325.

(1964): Studien zur Ökologie und Verbreitung der Gattung Chappuisius Kiefer 1938 (Copepoda, Haptacticoida); Mitteilung über Neufunde aus den Grundwasserströmen von Lahn, Niederrhein, Ruhr, Leine und Unterweser. - Crustaceana 6: 179-194.

(1966): Versuch einer ökologischen Gliederung des interstitiellen Grundwassers in Lebensbereiche eigener Prägung. - Arch. Hydrobiol. 62: 231-268.

(1966a): Die Organismengemeinschaften der Sandlückensysteme in natürlichen Biotopen und Langsamsandfiltern. - Veröff. d. Hydrol. Forsch. Abt. d. Dortmunder Stadtwerke AG 9: 93-113.

(1968): Langsamfilter als Biotopmodelle der experimentalökologischen Grundwasserforschung. - Gewässer u. Abwässer 46: 20-49.

(1968a): Ökologie, Systematik und Verbreitung zweier in Norddeutschland sympatrisch lebender Bathynella-Arten (Crustacea, Syncarida): - Int.J. Speleol.3: 111-145.

(1971): Die gegenseitige Ergänzung theoretischer und angewandter Grundwasser-Limnologie; mit Ergebnissen aus Wasserwerken Wiesbadens. - In: Die Sicherstellung der Trinkwasserversorgung Wiesbadens, Stadtwerke Wiesbaden A.G., Wiesbaden 1971: 79-90.

(1971a): Eine neue Methode zur Entnahme von Interstitialwasser aus subaquatischen Lockergesteinen. - Arch. Hydrobiol. 68: 519-527.

JAAG, O. (1952): Die Verschmutzung der Oberflächenwässer, eine Gefahr für das Grundwasser. - Schweiz. Ver. Gas- u. Wasserfachmännern, Monatsbulletin 6: 199-206.

LIEPOLT, R. (1968): Über den Chemismus des vertikal geschichteten Grundwassers dreier Tiefbrunnen im südlichen Wiener Becken. - Wasser u. Abwasser, 1968: 141-152.

NÖRING, F., FARKASDI, G., GOLWER, A., KNOLL, K. H., MATTHESS, G. \& SCHNEIDER, W. (1968): Über die Abbauvorgänge von Grundwasserverunreinigungen im Unterstrom von Abfalldeponien. - Das Gas- u. Wasserfach 6: 137-142.

PREUL, F. (1962): Gutachtliche Stellungnahme zur Frage einer Grundwassersenkung beim Wasserwerk der Stadt Fulda in der Johannesau. - Arch. d. Stadtw. Fulda, Fulda, Frankfurter Str. 7.

ROSSLER, B. (1951): Beeinflussung des Grundwassers durch Müll- und Schuttablagerungen. Vom Wasser 18: 43-60.

SOECKNICK, K., NUMANN, W., SARTORY, R., MALGRAS, J., ROMOND, Ch. \& HORB, B. (1970): Répartition des bactéries dans les sédiments du Lac de Constance. - Ann. Limnol. 6: 281-292.

ZWITTING, L. (1964): Die Beeinflussung des Grundwassers durch Mülldeponien. - Steir. Beitr. z. Hydrogeol. 1963/64: 91-101. 